Electron density measurement in atmospheric pressure plasma jets: Stark broadening of hydrogenated and non-hydrogenated lines

This content has been downloaded from IOPscience. Please scroll down to see the full text. 2015 Plasma Sources Sci. Technol. 24034001

(http://iopscience.iop.org/0963-0252/24/3/034001)

View the table of contents for this issue, or go to the journal homepage for more

Download details:

IP Address: 157.193.64.162

This content was downloaded on 20/04/2015 at 07:55

Please note that terms and conditions apply. 


\title{
Electron density measurement in atmospheric pressure plasma jets: Stark broadening of hydrogenated and non-hydrogenated lines
}

\author{
A Yu Nikiforov ${ }^{1,2}$, Ch Leys $^{1}$, M A Gonzalez ${ }^{3}$ and J L Walsh ${ }^{4}$ \\ ${ }^{1}$ Ghent University, Department of Applied Physics, Jozef Plateaustraat 22, B-9000 Ghent, Belgium \\ 2 Institute of Solution Chemistry RAS, Academicheskaya str, 1, 153045, Ivanovo, Russia \\ ${ }^{3}$ Universidad de Valladolid, Departamento de Fisica Aplicada, 47011 Valladolid, Spain \\ 4 The University of Liverpool, Department of Electrical Engineering \& Electronics, Brownlow Hill, \\ Liverpool, L69 3GJ, UK
}

E-mail: Anton.Nikiforov@Ugent.be

Received 4 November 2014, revised 17 February 2015

Accepted for publication 2 March 2015

Published 16 April 2015

\begin{abstract}
Electron density is one of the key parameters in the physics of a gas discharge. In this contribution the application of the Stark broadening method to determine the electron density in low temperature atmospheric pressure plasma jets is discussed. An overview of the available theoretical Stark broadening calculations of hydrogenated and non-hydrogenated atomic lines is presented. The difficulty in the evaluation of the fine structure splitting of lines, which is important at low electron density, is analysed and recommendations on the applicability of the method for low ionization degree plasmas are given. Different emission line broadening mechanisms under atmospheric pressure conditions are discussed and an experimental line profile fitting procedure for the determination of the Stark broadening contribution is suggested. Available experimental data is carefully analysed for the Stark broadening of lines in plasma jets excited over a wide range of frequencies from dc to MW and pulsed mode. Finally, recommendations are given concerning the application of the Stark broadening technique for the estimation of the electron density under typical conditions of plasma jets.
\end{abstract}

Keywords: plasma jet, electron density, stark broadening

(Some figures may appear in colour only in the online journal)

\section{Introduction}

Atmospheric pressure plasmas (APPs) are frequently used for both fundamental studies and in a variety of applications, ranging from materials processing to microbiology. In the last decade, many research teams have focused their efforts on the so-called atmospheric pressure plasma jet (APPJ) due to its remarkable ability to generate a long effluent, which extends in to the ambient air and contains a wide variety of active species. Compared to other plasmas sustained in a limited interelectrode space, plasma jets have the significant advantage of being able to transport reactive species to a remote region for treatment $[1,2]$. Up to now, many types of cold APPJs have been developed, such as those created by dc voltage, ac voltage with a frequency up to $100 \mathrm{kHz}, \mathrm{RF}$ power in the frequency range of 1 to $50 \mathrm{MHz}$, microwave, as well as plasmas generated by high voltage pulses of sub-nanosecond duration [3-7]. In view of the purpose of these plasmas to produce a large flux of radicals and other active species, it is important to understand the discharge properties; specifically, the electron density and temperature $\left(n_{e}\right.$ and $\left.T_{e}\right)$. Knowledge of plasma parameters and especially electron density is also a crucial point in validation of plasma models, where $n_{e}$ is usually the main input parameter [7]. The plasmas produced 
by such sources tend to exhibit similar proprieties, specifically, a low electron density $10^{10}-10^{13} \mathrm{~cm}^{-3}$ and a relatively high electron temperature. Driven by the need to develop ever more portable plasma sources for biomedical applications that are able to operate in molecular and reactive gases researchers are beginning to consider the use of micro-sized plasmas. Such discharges can exhibit electron densities in the range of $10^{14}-10^{18} \mathrm{~cm}^{-3}$ with gas temperatures exceeding $1000 \mathrm{~K}[8-10]$.

Atomic line Stark broadening, typically applied to the hydrogen Balmer series, is one of the most widely used techniques for $n_{e}$ measurement when considering APPJs. The method is popular since it gives a straightforward relation between the line width caused by Stark broadening and $n_{e}$ $[11,12]$. Usually, this method is applied for relatively dense plasmas, with electron density in the range $n_{e}>10^{14} \mathrm{~cm}^{-3}$ [13]. In [14] tables of hydrogen Balmer lines for plasma diagnostics were extended down to $10^{13} \mathrm{~cm}^{-3}$, but special care has to be paid to the simulation of spectral line fine structure at low densities which can add complexity to the analysis. Moreover, problems can occur if the gas density is high, such as the case at atmospheric pressure, as the van der Waals broadening can mask the Stark broadening and a rigorous deconvolution procedure has to be applied [15].

In this contribution, the use of atomic line Stark broadening for the measurement of $n_{e}$ in atmospheric pressure plasmas is considered. Applicability of the method for the analysis of APPJs is discussed in chapter 2. An overview of the various Stark broadening models available is given in chapter 3 . A deconvolution procedure for the estimation of resonance, van der Waals, Doppler, instrumental and Stark contributions in a spectral line profile is provided in chapter 4 with a focus on high electron density discharges. A view on the current progress in electron density diagnostics for APPJs is completed by considering non-hydrogenated line Stark broadening, which is important when hydrogenated lines are not present in the discharge or the electron density is very high. In chapter 5 new results of Stark broadening calculations are given which take into account fine structure of lines (FSL), in order to extend previously available results [14] to the case of low electron density plasmas. Finally, recommendations are provided for electron density measurements in APPJs with conclusions on the Stark broadening method.

\section{Methods for the measurement of electron density}

Techniques to determine the electron density can roughly be divided into electrical diagnostics and optical diagnostics. The first category comprises the various probe techniques (single, double electrical) and measurements of electron density from the current density. The second category can be subdivided into active and passive spectroscopy. Active spectroscopy is mostly based on the use of an external light source. Especially important are laser scattering experiments, particularly Thomson scattering (TS) which is a relatively straightforward technique allowing simultaneous determination of the main plasma parameters: $n_{e}$ and $T_{\mathrm{e}}$. In passive spectroscopy, the electron density can be obtained by the analysis of intensities and line shapes. An overview of the most common techniques for the measurement of $n_{e}$ in low temperature APPJs follows.

\subsection{Electrical probes}

The theory of probe measurements was first established in the 1920s by Langmuir for a case of low pressure plasma in a collisionless regime [16]. Recently a lot of effort has focused on adapting probe theory for $n_{e}$ measurements under the highly collision conditions, such as those found in atmospheric pressure plasma [17-19]. Tichy and co-workers [18] estimated $n_{e}$ to be about $1.7 \times 10^{13} \mathrm{~cm}^{-3}$ in a Radio Frequency $13.56 \mathrm{MHz}$ plasma jet working in He from the saturated ion current. The electron density estimation agrees well with the value obtained from the discharge resistivity. It has to be mentioned here that, even though there are some works related to the application of probes in high pressure plasmas, at the present moment the use of electrical probes to diagnose APPJs is not well established due the fact that information from the probe is strongly affected by collisions of charged particles in the probe sheath $[18,19]$.

\subsection{Current and voltage}

In the case of homogeneous plasma the current passing through the plasma cross-section can be presented as [20]:

$$
J=n_{e} e v_{e}\left(\frac{E}{N}\right)=n_{e} e \mu_{e} E
$$

where $J$ is the current density in $\mathrm{Am}^{-2}, e$ is the elementary charge in C, $E$ is the electrical field in $\mathrm{Vm}^{-1}$ and $\mu_{e}$ is the electron mobility of in $\mathrm{m}^{2} \mathrm{Vs}^{-1}$ and $v_{e}$ is the drift velocity of electrons in $\mathrm{ms}^{-1}$. The method is only valid for homogeneous plasmas; critically, in APPJs estimation of the plasma crosssection can be very difficult due to the complex geometry of the jet configuration or the formation of filaments. In [21] an Ar dielectric barrier discharge jet operating at $12 \mathrm{~W}$ was considered, the jet cross-section was that of a single filament and $n_{e}$ was found to be $1.5 \times 10^{13} \mathrm{~cm}^{-3}$. A similar approach to estimate the current density was used in [22] where a $30 \mathrm{~W}$, $350 \mathrm{kHz}$ discharge, formed of $245 \pm 21 \mu \mathrm{m}$ filaments, was found to have an electron density inside of the channels of approximately $9.9 \times 10^{15} \mathrm{~cm}^{-3}$. Using the current density method to establish the electron density is clearly indicative and has numerous pitfalls. In fact, in pulsed APPJs estimation of plasma cross-section is almost impossible due to the complex temporal and spatial behaviour of the discharge, with the formation of so-called 'plasma bullets' $[23,24]$. Additionally, the electron drift velocity $v_{e}$ strongly depends on the gas phase composition, see figure 1 . The gas composition varies significantly along the axis of the APPJ due to air diffusion and the air mole fraction can reach $10 \%$ on a distance of $10 \mathrm{~mm}$ from the nozzle [25] which can result in serious errors in the estimations of $n_{e}$ by the use of equation (1). 


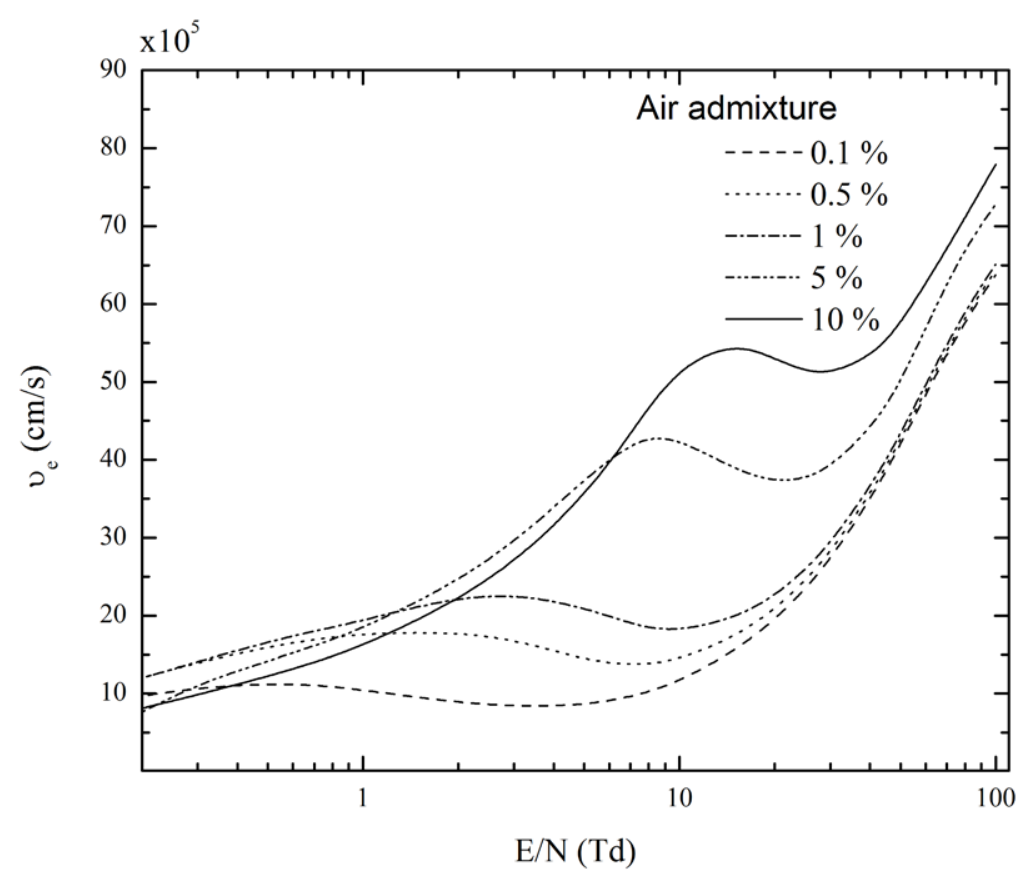

Figure 1. Drift velocity of electrons in Ar and Ar/Air mixtures for reduced field of 0.1-100 Td. Gas temperature $300 \mathrm{~K}$, pressure $760 \mathrm{Torr}$. Results of calculations are based on solution of Boltzmann equation with EEDF software [26].

\subsection{Infrared heterodyne interferometry}

Heterodyne interferometry is based on the measurement of the plasma refractive index which is related to a change of neutral and electron density in the discharge. In the method, the probing laser beam with frequency $\omega$ is separated into two paths, where the frequency in one of the paths is shifted to $\omega$ $+\Delta \omega$. First beam with frequency $\omega$ is passed through the discharge region where a phase shift is appeared due to a change in refractive index of the medium. After two beams merging at the interferometer, a phase shift $\Delta \Phi$ between the two beams can be measured by the heterodyne technique [27, 28]. The method is sensitive to change in density of both heavy particles and electrons. Obviously, there is a requirement to separate the two contributions which imposes limitations on the method and the technique itself can only be used in pulsed plasmas where a fast change of electron density and a slow change in heavy particles density are presented. In pulsed plasmas the phase shift due to electrons $\Delta \Phi_{e}$ can be measured experimentally and is directly related to the density, expressed by [29]:

$$
\Delta \Phi_{e}=-\frac{e^{2} \lambda}{4 c^{2} m_{e} \varepsilon_{0} \pi} n_{e} l
$$

where $e$ and $m_{e}$ is the electron charge and the mass, respectively, $c$ is a speed of light, $\varepsilon_{0}$ states for the vacuum permittivity, $\lambda$ is a wavelength of the source light and $l$ is the plasma length. The infrared heterodyne interferometry has been used in a micro-hollow cathode jet and in a pulsed dc jet where the peak electron density of $3.3 \times 10^{14} \mathrm{~cm}^{-3}$ and $2 \times 10^{14} \mathrm{~cm}^{-3}$ have been obtained respectively [28]. Schoenbach et al [27] applied heterodyne interferometry to microhollow cathode discharge in air and estimated electron density to be about $10^{13} \mathrm{~cm}^{-3}$. The results of laser heterodyne interferometry are found to be in good agreement with other methods with a very high limit of detection $1 \times 10^{12} \mathrm{~cm}^{-3}$ [29].

\subsection{Continuum radiation}

Measurements of continuum radiation have been used in several studies [30-32] in order to determine the electron density. The continuum radiation in plasma is generated by collisions of free electrons with atoms, ions and free-bound radiation. Due to the low degree of ionization in APPJs most of the contribution to the continuum is generated by electron-atom collisions [33]. The case of electron-atom collisions has been considered in [33] and an equation for $n_{e}$ for the case of low ionization degree $<10^{-5}$ has been obtained as follows:

$$
n_{e}=j_{e a}(\lambda) / g\left(\lambda, T_{e}\right) n_{a}
$$

Where $n_{a}$ is the neutral density, $j_{e a}(\lambda)$ in $\mathrm{Wm}^{-4} \mathrm{sr}^{-1}$ is the emission coefficient of continuum radiation due to electronatom collisions and $g\left(\lambda, T_{e}\right)$ can be expressed as:

$$
\begin{aligned}
g\left(\lambda, T_{e}\right)= & \frac{c_{1}}{\lambda^{2}} T_{e}^{3 / 2}\left\{Q\left(T_{e}\right)\left(1+\left(1+\frac{h c}{\lambda k_{\mathrm{B}} T_{e}}\right)^{2}\right)\right. \\
& \left.\times \exp \left(-\frac{h c}{\lambda k_{\mathrm{B}} T_{e}}\right)\right\}
\end{aligned}
$$

With $C_{1}=1.026 \times 10^{-34}\left(\mathrm{Jm}^{2} \mathrm{~K}^{3 / 2} \mathrm{~s}^{-1} \mathrm{sr}^{-1}\right), Q\left(T_{e}\right)$ is the mean cross section for momentum transfer of electrons [34] in $\mathrm{m}^{2}$ and $T_{e}$ in $\mathrm{K}$. The method requires absolute calibration of the optical system in order to obtain a value for $j_{e a}(\lambda)$. Recently the method was applied to a MW plasma jet working in Ar and it was found that in the core of the jet the electron density is about $10^{16} \mathrm{~cm}^{-3}[35]$. 


\subsection{Thomson scattering}

Thomson scattering is the scattering of light from free charged particles in the plasma, which are predominantly electrons, it is one of the few techniques that enables the direct and simultaneous measurement of electron density and electron temperature [36]. According to the theory, electron density can be determined through measurement in absolute units of the scattered power $d P_{s}$ arrived at the detector by following relation [36]:

$$
d P_{s}=P_{i} n_{e} \sigma_{d}^{T} L S\left(\vec{k}, \omega_{F}\right) \mathrm{d} \Omega
$$

where $P_{i}$ the incident laser power, $\sigma_{d}^{T}$ the differential cross section for Thomson scattering, $L$ the length of the detection volume along the laser path, $S\left(\vec{k}, \omega_{F}\right)$ the so-called dynamic form factor, and $\mathrm{d} \Omega$ the solid angle of detection. The theory of Thomson scattering is well developed because of the wide use of the method in high temperature, high density plasmas $[37,38]$. In low density plasmas, the method can prove expensive as it demands the use of a triple grating monochromator for the subtraction of stray light and the Rayleigh scattering component. Thomson scattering is capable of measuring the electron density to the limit as low as $5 \times 10^{12} \mathrm{~cm}^{-3}$ with high spatial resolution up to $\mu \mathrm{m}$ scale. Sadeghi and co-workers [39] have used the Thomson scattering method for electron density measurements in an Ar low current micro-discharge. Electron density was estimated to be $(6 \pm 3) \times 10^{13} \mathrm{~cm}^{-3}$ and $3 \times 10^{5}$ laser pulses were used for signal accumulation. Recently Thomson scattering experiments have been carried out on an APPJ sustained by MW power [40]. The problem of disentangling the contributions of Thomson scattering on electrons and Raman scattering on molecules of $\mathrm{N}_{2}$ and $\mathrm{O}_{2}$, appearing due to air diffusion into the jet, was overcome by a newly designed fitting procedure described in [40]. It was found that Thomson scattering diagnostics can be applied up to $1 \%$ admixture of air into the plasma working gas, which can be a limit of the method for APPJs diagnostics.

\subsection{Microwave radiation scattering}

The measurements of radiation scattering from plasmas irradiated with microwaves (MW) has been proposed theoretically as a new method to estimate electron density in 2005 by Shneider and Miles [41]. The MW radiation scattering can be treated in a similar way to Rayleigh scattering of light in a case of micro-plasmas having transversal size and skin-layer thickness much less than wavelength of the microwave radiation $\lambda_{\mathrm{MW}}$. According to [41-43] microwave scattering from the plasma can be calculated by following:

$$
E_{\text {peak }} \propto E_{m 0} n_{e}(t)
$$

where $E_{\text {peak }}$ is the time dependent electric field of the scattering signal, $E_{m 0}$ is the incident microwave electric field, and $n_{e}(t)$ is the time varying number of electrons in the plasma. The coefficient of proportionality in equation (6) can be found through calibration of the MW scattering system with dielectric scatterers of well-known properties. In [42] dielectric materials: Teflon $(\varepsilon=2.1)$, alumina $(\varepsilon=9.2)$, polyethylene $(\varepsilon=2.25)$, and quartz $(\varepsilon=3.8)$ were proposed as calibrators of the MW system. The method was used to study a He plasma jet sustained by an ac voltage of $3.8 \mathrm{kV}$. It was found that after discharge initiation the plasma density reaches about $5-10 \times 10^{13} \mathrm{~cm}^{-3}$ and then decays with characteristic times of a few microseconds. The MW method was used in [43] to measure the temporal evolution of electron density with $\mu \mathrm{s}$ resolution in a similar He DBD jet. The discharge was driven by an ac high voltage source with a discharge voltage of several $\mathrm{kV}$ and a frequency of $15-30 \mathrm{kHz}$. The plasma density was found to be in the order of $10^{13} \mathrm{~cm}^{-3}$ which is in good agreement with other methods. MW scattering can be applied to plasmas of low ionization degree with a minimum $n_{e}$ of approximately $10^{9} \mathrm{~cm}^{-3}$ and shows possibilities of obtaining the decaying plasma parameters and information about loss rates of electrons $[41,44]$. It has to be noted here that a drawback of the method is that only volume averaged value of $n_{e}$ can be measured.

\subsection{Broadening of emission lines}

Analysis of the Stark broadening of spectral lines emitted by the plasma is the most used method to measure the electron density $[11,12]$. From a technical point of view the method is much more affordable then either the Thomson scattering or laser heterodyne interferometry techniques and can provide information on electron density with high accuracy. In many works, hydrogen lines are the first choice for plasma diagnostic applications because of the linear Stark effect and position in visible spectral region where high sensitivity and spectral resolution can be achieved with commercially available spectrometers. Typically, the main limitation of the Stark broadening method is that most of the theoretical expressions for $n_{e}$ are applicable only for dense plasmas with a lower limit for $n_{e}$ in the range of $10^{16}-10^{17} \mathrm{~cm}^{-3}$ [12] with an error of about $7 \%[13,45]$. Extension of the method to low ionization degree, high pressure plasmas, like APPJs with an electron density in the range of $10^{13} \mathrm{~cm}^{-3}-10^{15} \mathrm{~cm}^{-3}$ requires consideration of the fine structure of the line (for densities below $10^{14} \mathrm{~cm}^{-3}$ ) and a rigorous deconvolution of the line profile so the Doppler, van der Waals, and resonance components can be estimated, enabling the Stark contributions to be found.

Table 1 provides an overview of all methods applicable to measure electron density in APPJs, highlighting their limitations in terms of spatial and temporal resolution. In the following chapter a short overview of Stark broadening theory is given with analysis of the theory limits.

\section{Overview of Stark broadening theory and application}

It is well known that plasma consists of both charged and neutral particles; yet, on a macroscopic scale they can be considered neutral. Due to the distribution of the particles in the plasma, atoms at different points in the discharge experience different electric fields which may fluctuate in time, due to the relative movement between atoms and the charged particles 
Table 1. Overview of the methods applicable to measure electron density in atmospheric pressure plasmas.

\begin{tabular}{|c|c|c|c|c|c|}
\hline Method & $\begin{array}{l}N_{e} \text { limit } \\
{\left[\mathrm{cm}^{-3}\right]}\end{array}$ & Spatial resolution & Temporal resolution & Advantages/Disadvantages & Ref. \\
\hline Electrical probes & $10^{9}$ & $\begin{array}{l}\text { mm range, depends on } \\
\text { probe size }\end{array}$ & ms range & $\begin{array}{l}\text { Theory does not exist for high } \\
\text { pressure case }\end{array}$ & [16-19] \\
\hline Current and voltage & any & $\begin{array}{l}\text { Cross-section } \\
\text { integrated }\end{array}$ & $\begin{array}{l}\text { Up to ns, depends on } \\
\text { plasma }\end{array}$ & $\begin{array}{l}\text { Only rough quasi-quantitative } \\
\text { estimation of } n_{e} \text {. }\end{array}$ & [20-25] \\
\hline $\begin{array}{l}\text { Infrared heterodyne } \\
\text { interferometry }\end{array}$ & $10^{12}$ & $\begin{array}{l}\text { Up to } \mu \mathrm{m} \text {, depends on } \\
\text { beam size }\end{array}$ & $\mu$ s range & $\begin{array}{l}\text { Only applicable to pulsed plasmas. } \\
\text { Line integrated value. Relatively } \\
\text { expensive. }\end{array}$ & [27-29] \\
\hline Continuum radiation & $10^{13}$ & Volume integrated & ns range & $\begin{array}{l}\text { Requires absolute calibration of the } \\
\text { spectrometer }\end{array}$ & [30-35] \\
\hline Thomson scattering & $10^{11}$ & $\begin{array}{l}\text { Up to } \mu \mathrm{m} \text {, depends on } \\
\text { beam size }\end{array}$ & Up to ps & $\begin{array}{l}\text { Direct measurements of } n_{e} \text { and } T_{e} \text {. } \\
\text { Very sophisticated and expensive. }\end{array}$ & [36-38] \\
\hline $\begin{array}{l}\text { Microwave radiation } \\
\text { scattering }\end{array}$ & $10^{12}$ & Volume averaged & $\mu$ s range & Requires absolute calibration & [41-43] \\
\hline $\begin{array}{l}\text { Broadening of emission } \\
\text { lines }\end{array}$ & $10^{13}$ & Line of sign integrated. & Up to ps & $\begin{array}{l}\text { Can requires sophisticated analysis } \\
\text { in case of low } n_{e}\end{array}$ & [11-15] \\
\hline
\end{tabular}

surrounding them. This perturber electric field changes the eigenvalues of the energies of the atoms, breaking partially the degeneracy of the atomic levels, what is known as the Stark effect [46]. This field also affects the selection rules of the optical transitions between two states of each atom [47]. Considering the distribution of emitters and charged perturbers in the plasma, the collective consequence of those changes on the observed spectral lines emitted results in a variation of the line shape, width and position depending on the plasma electron density and temperature. For many conditions of electron density and temperature the number of charged particles in the plasma is much higher than the number of neutrals; under this condition the interactions suffered by the atoms are dominated by the Stark effect, what makes the analysis of Stark broadened line profiles an ideal tool for plasma diagnostics.

The interactions between charged perturbers in the plasma and emitters were described classically using two models, quasistatic broadening for the heavy ions and the impact broadening for the fast moving electrons. For the quasistatic broadening, the characteristic frequencies of the electric field evolution are very small in the time scale of the atom optical emission, thus the movement of the perturbers can be neglected [12]. Under this assumption the line profile can be obtained as an average over the profiles corresponding to different static configuration of the heavy perturbers, weighted according to the probability of each configuration. One of the most successful probability field distribution models used for these calculations is described in [48]. On the other hand, impact approximation is based on the assumption that the correlation time for the dipole moment of the emitter is much longer than the typical duration of the collisions between the emitter and the fast perturbers, such that the collisions can be considered as instantaneous from the emitter's point of view. These collisions are also considered independent and typically weak (in terms of the change produced in the emitter wave function) meaning the important parameter to obtain the line broadening is the frequency of the collisions [12]. Based on this assumption, a broadened line profile for an atom in a plasma with fast (impact) electrons and much heavier (static) atoms can be obtained, assuming statistical independence ([49] provides an interesting analysis about this approximation) between both mechanisms, as the convolution of both effects:

$$
I(\omega)=\int W(E) I(\omega, E) \mathrm{d} E
$$

where $I(\omega, E)$ is the broadened profile due to the impact electrons for a given ionic field $E$ with statistical weight $W(E)$. From the point of view of the practical use of Stark broadened profiles for plasma diagnostics, some of the works based on the model described above led to the production of tables of hydrogen Stark broadened line profiles by Vidal, Cooper and Smith [50], later extended in [51], and also the tables of Stark broadening parameters and profiles in [12], work that is still a reference for researchers in plasma spectroscopy.

However this relatively simple technique soon showed discrepancies when compared against carefully conducted experimental studies. The cause proposed as the origin of these discrepancies was the so-called ion-motion effect [52], now usually called ion-dynamics, what was soon confirmed by experiments on the line broadening in plasmas with ionic perturbers of different masses [53-56]. Then, important efforts were devoted to reduce those discrepancies and, as a consequence, different models or calculation methods were developed to consider ion-dynamics in the line broadening calculations. Three of the more successful approaches will be briefly described here, the model microfield method (MMM), the computer simulation method (CSM) and the frequency fluctuation method (FFM). It is interesting to note that in spite of the important work developed along many decades, discrepancies still remain between the results obtained using the different methods [57].

The MMM, based on statistical approximations [58-60], describes the electric field experienced by the atoms in a plasma using a Markovian process. In this process the microfield is considered constant along given time intervals and then changes instantaneously to a different constant value for another time interval. The time interval jumps follow a 
Poisson law and the jumping frequencies are chosen to reproduce the field autocorrelation function. The same process, with different characteristic parameters, is used for the electron and ion fields in the plasma. The MMM was used to obtain profiles of the hydrogen Lyman-alpha and Balmer-alpha and -beta lines [61] and to analyze ion-dynamic effects in them [62]. More recently the MMM has been used to obtain extensive tabulations of Stark broadened hydrogen line profiles for plasma diagnostics $[63,64]$. The agreement of the MMM calculations with the experimental profiles noticeably improved that of previous calculations due to its consideration, at least in an approximate way, of the ionic movement. One interesting and innovative contribution of the MMM to the development of other line shapes calculation methods was its 'field-point of view', what was later used by computer simulations. A good review of this model and its validity can be seen in [65].

The first paper on the calculation of line shapes using CSM was a work fully applied to analyze the ion-dynamics effects on the hydrogen Lyman-beta line [66]. For this, the movement of ionic perturbers with different masses was reproduced in the computer and the results showed good agreement with the available experimental data [67]. In that first work the relative movement between the emitter and the heavy perturbers was described using the so-called $\mu$-ion model, whose validity was later studied in another work [68] and is still a common method used in many simulation techniques, including those whose results are shown later in this contribution. The good agreement between these simulations and the experiments gave rise to a growth in the number of research groups studying line broadening using simulation techniques [69-77]. Computer simulations have been used as the basis of computer programs for electron density determination from experimental profiles [78] and to obtain large tabulations of Hydrogen [14, 79] or Helium Stark profiles [80, 81]. CSM enables line profiles for a wide range of plasma densities, temperatures and compositions to be obtained [82], as well as for cases with plasma unbalances or inhomogeneities that can occur in experimental studies. The profiles obtained considering specific experimental conditions allow researchers to undertake plasma diagnostics by direct comparison of the line profiles obtained in the simulations and those experimentally recorded. More details on CSM for line shapes calculations can be found in the review [83].

Despite their versatility and general good degree of accuracy, computer simulations have an important drawback: to obtain a spectral profile using computer simulation necessitates a large amount of calculation, meaning simulations can take far longer to obtain line profiles than analytical or approximate methods. Consequently, alternative methods that are computationally less expensive have recently been developed in order to be able to supply line profiles for immediate comparison with experimental results. The FFM [84] is perhaps the best of these approximate methods as its results have been successfully compared with those obtained using simulation methods for different spectral lines and plasma conditions $[57,85,86]$; additionally, it readily enables other phenomena to be considered in the calculations, such as radiation redistribution in the plasma [87] or the Zeeman broadening [88, 89].
Furthermore, it has also been the basis of other calculation methods, such as the Quasicontiguous frequency (QC) FFM [90] used to obtain analytically high-n $n>1$ transitions which are typically cumbersome to simulate. The FFM permits researchers to obtain good quality complex line profiles with calculation times shorter than those needed for computer simulations, without sacrificing data quality. Briefly, the FFM considers the usual separation of the line broadening into the homogeneous broadening effect of the impact electrons and the inhomogeneous broadening effect arising from the slow ions and a component mixing effect due to ion dynamics through a Markov process.

When comparing calculated line profiles with the profiles recorded experimentally we must remember that, unlike in a computer simulation, in the real experiments it's often impossible to turn on or off different effects at demand; subsequently, the recorded line shapes are the results of many different phenomena. Typically, line shapes emitted by an atom or ion in a plasma suffer broadening and shifting either due to the interaction of the emitter with other particles in the plasma, what is usually called the pressure-broadening that includes Stark, van der Waals and resonance broadening, or to the movement of the emitters in the plasma, or even to the usually least important of all, the natural broadening. The plasma conditions, electron density, collider nature, electron and gas temperature and pressure determine the relative weight of each contribution in the broadening of the emitted line. It is also important to note that for the same plasma conditions the relative importance of those broadening mechanisms can be different for different lines. We must also consider how other influences, such as the self-absorption of the line as the emitted light propagates within the plasma and the instrumental broadening, can affect the recorded shape of the line. As such, in order to extrapolate accurate diagnostic information from the recorded line profiles, an evaluation of the importance of the different effects, or even correction, must be performed, this process is discussed in detail in several recent reviews [15, 91-94] and in the context of APPJ's in this review.

Considering the case of atmospheric pressure plasmas the broadening of the line will be a combination of the following phenomena:

- Instrumental broadening

- Natural broadening

- Doppler broadening

- Pressure broadening which is a combination of: - van der Waals broadening

- resonance broadening

- Stark broadening

\section{- Self-absorption broadening}

Other broadening mechanisms, for example the Zeeman effect, aren't usually present in atmospheric pressure plasma jets and so they are not discussed here. However, for a case in which other broadening phenomena are present, it's interesting to notice that different calculation methods, such as computer simulation or the FFM mentioned before, allow the broadened line profiles to be obtained when the emitter 
is simultaneously undergoing several broadening mechanisms $[88,89,95]$. Depending on the plasma conditions an estimation of the Stark component can be a non-trivial task. The choice of the deconvolution procedure as well as uncertainty of the final result strongly depends on the ratio of the Stark component to all others. In the next section we will consider two cases: atmospheric pressure plasmas jets of high electron density of $n_{e}>10^{14} \mathrm{~cm}^{-3}$ with a typical example of a ns pulsed discharge and low electron density APPJs with $n_{e}<10^{14} \mathrm{~cm}^{-3}$.

\section{High electron density discharges}

The deconvolution of the various contributions in the line broadened profile can be carried out based on the fact that, in many cases, the experimentally measured line profiles can be fitted with the Voigt function which is the convolution of the two functions: Gaussian and Lorentzian with the full width at half maximum (FWHM) given by $\Delta \lambda_{1 / 2}^{G}$ and $\Delta \lambda_{1 / 2}^{L}$. The Voigt function can be represented through the combination of the Gauss and Lorentz components as follow [96]:

$$
\begin{aligned}
V(x, a) & =\frac{a}{\pi} \times \int_{-\infty}^{+\infty} \frac{\mathrm{e}^{-t^{2}}}{a^{2}+(x-t)^{2}} \mathrm{~d} t \\
x & =\frac{\lambda-\lambda_{0}}{\Delta \lambda_{1 / 2}^{G}} \times 1.665 \\
a & =\frac{\Delta \lambda_{1 / 2}^{L}}{\Delta \lambda_{1 / 2}^{G}} \times 0.83255
\end{aligned}
$$

where $x$ and $a$ are dimensionless parameters and $\lambda_{0}$ is the center wavelength of the line in $\mathrm{nm}$. The broadening contributions with a Gaussian shape will lead to a profile with $\Delta \lambda_{1 / 2}^{G}$ determined as:

$$
\Delta \lambda_{1 / 2}^{G}=\left(\sum_{i}\left(\Delta \lambda_{1 / 2}^{G_{i}}\right)^{2}\right)^{0.5}
$$

Whereas for Lorentzian broadening components the final contribution to the line profile will also be Lorentzian, with FWHM determined as:

$$
\Delta \lambda_{1 / 2}^{L}=\sum_{j} \Delta \lambda_{1 / 2}^{L_{j}}
$$

Here we will give a short overview of the fundamental characteristics of each broadening mechanism with approximate formulae for evaluation of their line profile parameter $\Delta \lambda_{1 / 2}$. Further information on the theory of broadening mechanisms can be found in numerous books and articles $[11,12$, 97-99].

\subsection{Natural broadening}

Natural broadening arises due to the finite lifetime of the excited levels and is typically very small, on the order of $10^{-4} \mathrm{~nm}$ and can be neglected in the case of APPJs spectroscopy.

\subsection{Instrumental broadening}

Any optical instrument has its own resolution and emission lines will be affected by the instrument. In most spectrometers used for the diagnostics of APPJs the instrumental function has a Gaussian profile with FWHM $\Delta \lambda_{I}$ which can be easily measured using single mode laser or a low pressure lamp. In the case of a low pressure lamp, the emission lines are limited to a Doppler broadened profile with a very narrow FWHM of some pm. If the width of the Doppler profile is smaller than the instrumental width, then the measured profile of a lamp line using the instrument (the spectrometer) is a convolution of the instrumental profile and Doppler profile and can be precisely determined if gas temperature in the lamp is known. By subtraction of the Doppler contribution from the measured lamp profile in accordance with equations (8)-(12) the $\Delta \lambda_{I}$ can be determined. It is also worth noting that situations when the instrumental function is non-Gaussian can occur, e.g. if an interferometer is used to record the line profile. In this case equations (8)-(12) cannot be used and a full deconvolution of the line profile and the instrumental function has to be carried out prior to any other steps. The deconvolution of two profiles can be performed by using the Tikhonov regularization method with various freely available codes, see e.g. [100] and references therein.

\subsection{Self-absorption}

Self-absorption distorts and broadens spectral lines, this leads to an overestimation of the electron density calculated from the widths of self-absorbed lines if no correction of the recorded profiles is performed [91]. One very common technique is the use of a concave mirror behind the plasma to double the optical path length and record the signal intensity with and without mirror. As self-absorption becomes more important near the center of the line, where intensity is higher, a simple self-absorption test consists on measuring the proportion of intensities between both signals, with and without mirror, along the full profile. If the proportion gets smaller as we approach the line center then there is selfabsorption and the measured profile can be corrected if the optical depth is not large [101]. Optical thickness of the plasma can be tested as well by measurements of the ratio of line intensities within multiplet where states are differently affected by self-absorption [94]. Presence of self-absorption in the case of APPJs can be probed also by measurement of the line profile across and along of the plasma jet. In a typical plasma jet the difference in radial and axial dimensions can reach two orders of magnitude which leads to a stronger self-absorption along the jet yielding an effect similar to the concave mirror method. In strongly non-homogeneous APPJs the effect of self-absorption is very difficult to include in calculations, see e.g. [91, 102] and whenever it is possible atomic lines with low absorption coefficients have to be used in electron density measurements. It is also interesting to note that for lines with forbidden components the distance between the allowed and forbidden component depends on the plasma electron density while it is not 


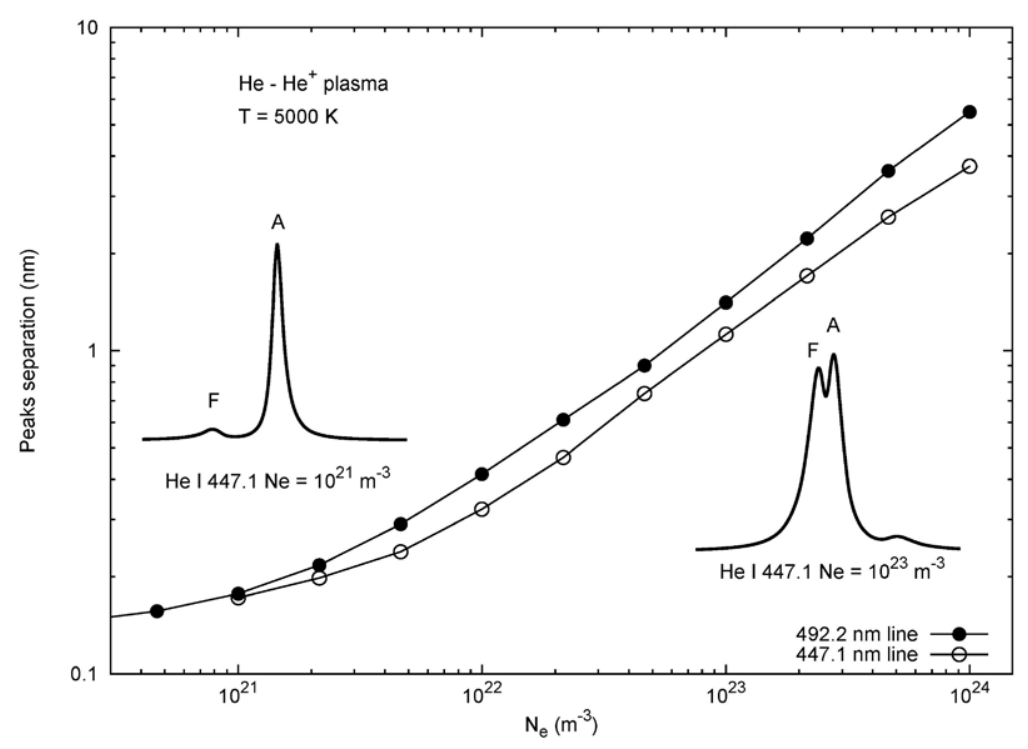

Figure 2. Distance between the allowed $(a)$ and the main forbidden $(f)$ component of the He I $447.1 \mathrm{~nm}$ and $492.2 \mathrm{~nm}$ lines. As this distance does not depend on the existence of self-absorption, it can be used as an alternative diagnostic method under those circumstances. The two line profiles included in the figure illustrate the difficulty of using this technique at low densities when the forbidden component is weak.

affected by self-absorption, hence plasma diagnostics can be done by measuring that distance without the problems derived of self-absorbed lines [92]. For example, for He I lines with forbidden components different works have performed plasma diagnostics by using approximate formulas [81, 103-105] or available tables of Stark broadened profiles $[80,81]$. One drawback of this technique comes from using lines with forbidden components where the forbidden component can have a very low intensity; at low electron densities (a little below $10^{15} \mathrm{~cm}^{-3}$ ) the intensity of the component can be masked by experimental noise or other lines [92]. Furthermore, at such low densities the allowed-forbidden distance barely changes with the electron density, as can be seen in figure 2; consequently, small experimental inaccuracies in the determination of the distance can produce large errors in the determination of the electron density. In order to see how the relative intensities of both, allowed and forbidden, components change with electron density, and the small intensity of the forbidden component at low values of $n_{e}$, two profiles of the $\mathrm{He} 447.1 \mathrm{~nm}$ line are shown as inserts in figure 2 , one corresponds to a relatively low density, $n_{e}=$ $10^{15} \mathrm{~cm}^{-3}$, and another to a higher value, $n_{e}=10^{17} \mathrm{~cm}^{-3}$.

\subsection{Doppler broadening}

The relative motion of an emitter to the detector leads to a shifted line. In plasma with gas temperature $T_{g}$ the line profile due to the presence of Doppler effects can be well described by a Gaussian profile with a FWHM (nm):

$$
\Delta \lambda_{D}=\lambda_{0}\left(8 \ln 2 \frac{k_{\mathrm{B}} T_{g}}{m_{a} c^{2}}\right)^{0.5}
$$

where $k_{\mathrm{B}}$ is the Boltzmann constant (unit: $\mathrm{JK}^{-1}$ ) and $T_{g}$ the gas temperature in $\mathrm{K}$, and $m_{a}$ the mass of the emitter.

\subsection{Resonance broadening}

Resonance broadening occurs for transitions involving a level that is dipole-coupled to ground state while emitters are surrounded by identical perturbers in the ground state. If $g$ stands for the ground state level of the radiative transition the evaluation formula for the FWHM (nm) of resonance broadening is given [106]:

$$
\Delta \lambda_{R} \cong \frac{3 e^{2}}{16 \pi \varepsilon_{0} m_{e} c^{2}} \lambda_{u l}^{2} \lambda_{l g} f_{g l} N_{g} \sqrt{g_{g} / g_{l}}
$$

where $e$ and $m_{e}$ are the electron charge (unit: C) and mass $(\mathrm{kg})$, respectively. $\varepsilon_{0}$ is the vacuum permeability $(\mathrm{F} / \mathrm{m})$ and $f_{g l}$ is the oscillator strength of the corresponding allowed transition between level $g$ and low energy excited level $l . g_{\mathrm{g}}$, $g_{l}$, are the statistical weights and $N_{g}$ is the density $\left(\mathrm{m}^{-3}\right)$ of the corresponding states. $\lambda_{u l}$ and $\lambda_{l g}$ stands for the line centre wavelength of the corresponding transition between upper $u$ and lower level $l$ and ground level. The resonance broadening has a Lorentzian profile. At atmospheric pressure resonance broadening for hydrogen Balmer lines is negligibly small and can be excluded from calculation. Nevertheless, the use of non-hydrogenated lines of $\mathrm{Ar}$ or He can require estimation of the resonance broadening contribution which can be comparable with other broadening components at atmospheric pressure [107].

\subsection{Van der Waals broadening}

Van der Waals broadening is a result of the dipole interaction between an excited radiator and the induced dipole of a neutral ground state particle with density $N_{g}$. According to the Lindholm-Foley theory [108] van der Waals broadening has a Lorentzian shape and the approximate expression for the FWHM (nm) can be given as: 


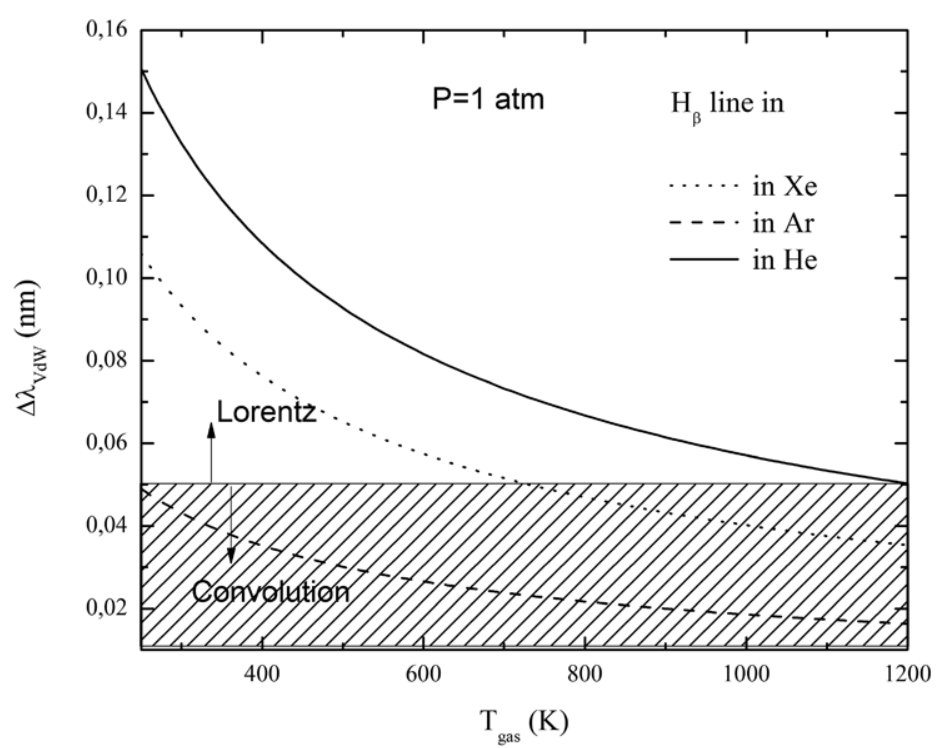

Figure 3. Van der Waals broadening of $H_{\beta}$ line at atmospheric pressure in different mixtures. Zone 'Lorentz' corresponds to conditions where the profile of the line can be approximated by a Lorentzian shape. 'Convolution' zone corresponds to the situation where fine structure of the $H_{\beta}$ line has to be considered.

$$
\Delta \lambda_{V}=\frac{\lambda_{u l}^{2}}{2 \pi c} \cdot 8.16\left(\frac{C_{6}}{\hbar}\right)^{2 / 5} \bar{v}^{3 / 5} N_{g}
$$

Where $\bar{v}$ is the average relative velocity between radiator and perturber

$$
\bar{v}=\left(\frac{8 k_{\mathrm{B}} T_{g}}{\pi \mu}\right)^{1 / 2}
$$

with the reduced mass $1 / \mu=1 / m_{a}+1 / m_{b}$.

In equation (15) $\mathrm{C}_{6}$ stands for the van der Waals constant related to the short-range interaction and can be measured or calculated as:

$$
C_{6}=e^{2} \alpha a_{0}^{2}\left[\overline{R_{u}^{2}}-\overline{R_{l}^{2}}\right]
$$

where $\alpha$ and $a_{0}$ are the average dipole polarizability of the perturbers and Bohr radius, respectively. $\overline{R_{u}^{2}}$ and $\overline{R_{l}^{2}}$ are the meansquare radii of levels $u$ and $l$ of the transition, respectively. $\overline{R_{u}^{2}}$ and $\overline{R_{l}^{2}}$ may be calculated from:

$$
\overline{R_{j}^{2}}=\frac{n_{j}^{*^{2}}}{2}\left[5 n_{j}^{*^{2}}+1-3 l_{j}\left(l_{j}+1\right)\right]
$$

with $l_{j}$ the orbital quantum number and $n_{j}^{*^{2}}$ the square of effective quantum number of level $j$, respectively.

$$
n_{j}^{*^{2}}=\left(\frac{E_{\mathrm{Ryd}}}{E_{\mathrm{ion}}-E_{j}}\right)
$$

where $E_{R y d}$ denotes the Rydberg constant, $E_{i o n}$ is the ionization level of the radiator, and $E_{j}$ the energy of the upper or lower level of the transition line. When considering the widely used hydrogen Balmer lines care is required in the calculation of $\Delta \lambda_{V}$ and the deconvolution of the van der Waals contribution considering all the line components. Following [109] for the Balmer beta line the total hydrogen line profile due to van der Waals broadening can be well approximated by the Lorenz profile if $\Delta \lambda_{V}>0.05 \mathrm{~nm}$ which is 5 times the separation between individual components of the unperturbed atom (the separation increases if an external field, fixed or due to the plasma, acts on the atom). Otherwise the fine structure of the line has to be taken into account and the calculated profile will have a non-Lorentzian shape. Figure 3 presents the van der Waals broadening of the $H_{\beta}$ line in atmospheric pressure conditions over a range of typical temperatures in Xe, Ar, and He. The region where the profile of the van der Waals component of the $H_{\beta}$ line is strongly affected by the fine structure of the line depends on the mixture and can be important even at low temperatures of $280 \mathrm{~K}$.

\subsection{Stark broadening}

Deconvolution of all broadening components from the experimentally measured profile in accordance with equations(8)-(12) enables the FWHM of the Stark broadening $\Delta \lambda_{S}$ to be determined. The scheme of the deconvolution procedure without taking into account self-absorption broadening is presented in figure 4. In plasma with a high electron density the Stark broadening $\Delta \lambda_{S}$ can be directly related to electron density and used for estimation of $n_{e}$. The hydrogen Balmer series is commonly used to measure electron density but this can often be problematic due to spectral overlap of neighboring emission lines in APPJs. Even in noble gas micro-plasmas it is not uncommon to observe emission lines produced from metal sputtered from the electrodes and subsequently excited. For example, Zhu et al [110] observed $\mathrm{Cr}(\mathrm{I})$ emission at $487.1 \mathrm{~nm}$, overlapping with the $H_{\beta}$ profile and Walsh et al [111] observed strong emission from both $\mathrm{Cr}(\mathrm{I})$ and $\mathrm{Fe}(\mathrm{I})$ making it impossible to determine the electron density from the $H_{\beta}$ emission line. Additionally, the presence of hydrogen in such APPJs can be minimal, resulting in low emission intensity from the Balmer series. Due to these difficulties there is a considerable 


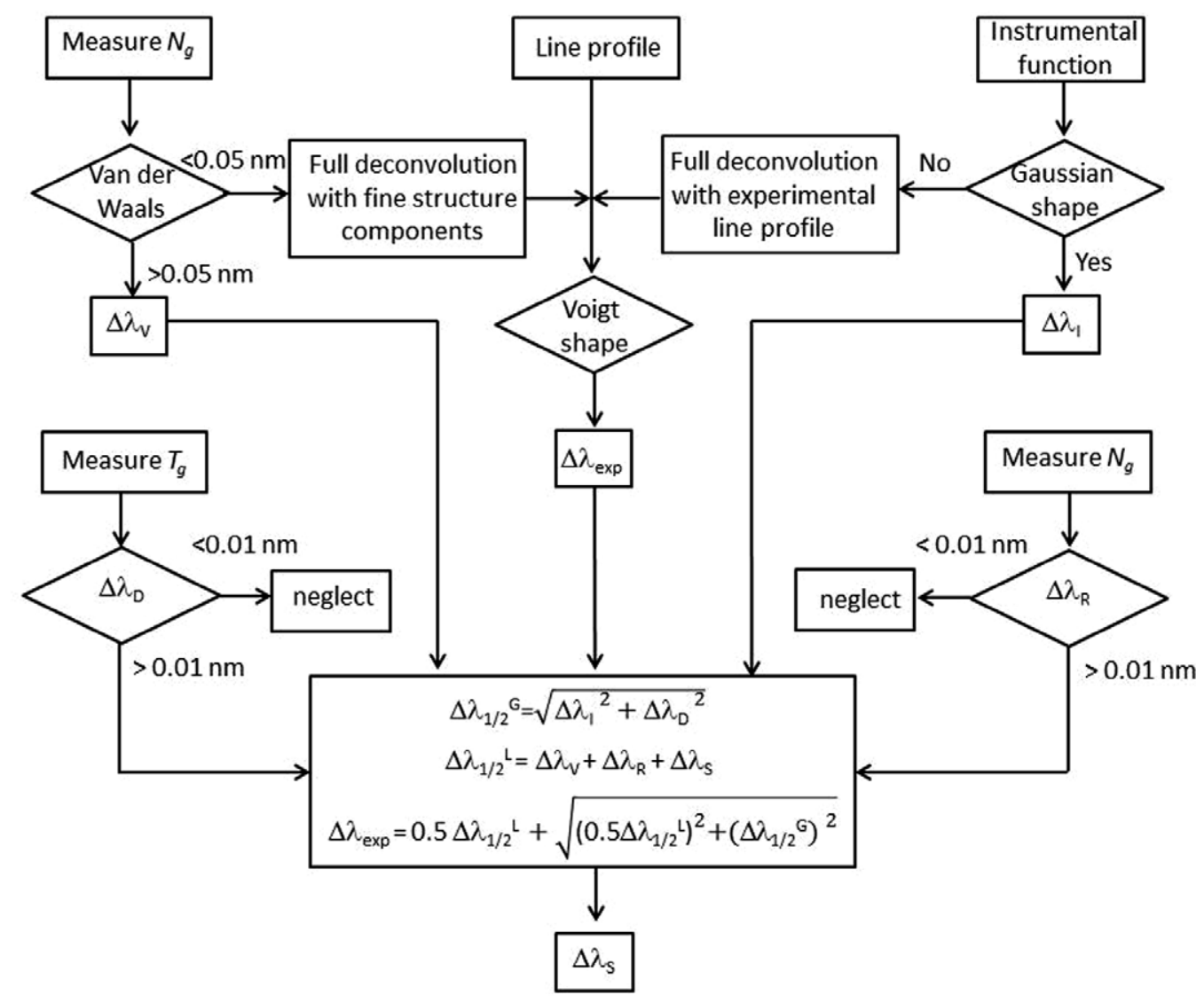

Figure 4. Scheme of the deconvolution procedure for estimation of Stark broadening contribution in a high electron density atmospheric pressure discharge.

interest in the use of Stark broadening of isolated non-hydrogenic lines from neutral atoms and positive ions to determine $n_{e}$. While the hydrogen Balmer series provides a sensitive indicator of electron density due to the large linear Stark effect; non-hydrogenic lines are considerably less sensitive due to the quadratic Stark effect exhibited [112]. Essentially, the use of non-hydrogenic lines to measure $n_{e}$ is practically limited to discharges where the density exceeds $5 \times 10^{15} \mathrm{~cm}^{-3}$ [113]. While traditional plasma sources used in biomedical applications, such as dielectric barrier jets, exhibit electron densities many orders of magnitude below this threshold, the recent advent of high-density micro-plasma sources renews interest in the use of non-hydrogenic lines for $n_{e}$ determination. The set of widely used empirical equations obtained theoretically and experimentally for lines of $\mathrm{He}, \mathrm{Ar}, H_{\beta}, H_{\alpha}, H_{\gamma}$, which are important in diagnostics of APPJs, are presented in table 2. Tabulated data for $\Delta \lambda_{S}$ for different temperatures and high electron densities of $10^{16}-10^{19} \mathrm{~cm}^{-3}$ are available also as Griem tables in $[11,12]$ for hydrogen as well for Ar and He. The authors of [14] provided two different line widths versus electron density fittings: on the one hand the usual line width at half maximum, and on the other hand the line width at half area. The fitting equations for $H_{\alpha}$ and $H_{\gamma}$ in table 2 and [11] are presented for Stark broadening at full width at half area $\left(\Delta \lambda_{s}^{A}\right)$ which is different from $\Delta \lambda_{S}$. These fittings were derived from those originally calculated in [14] with the only difference of correcting the mistyping in [14], where it should have indicated HWHM instead of FWHM. In [14] it was shown that $\Delta \lambda_{s}^{A}$ is less sensitive to the ion dynamics in comparison to $\Delta \lambda_{S}$ and can be considered as more appropriate for calculations of $n_{e}$ in discharges with a low ionization degree. This is especially important for the $H_{\alpha}$ and $H_{\gamma}$ as their peak intensities, and consequently their widths at half maximum, are strongly affected by ion dynamics effects. However this effect has a nearly negligible influence on the $H_{\beta}$ width.

Theoretically calculated profiles, fitting equations and tabulated data are extensively used in the analysis of plasmas related to biomedical applications. In [120] an ac discharge generated with frequencies from 10 to $42 \mathrm{kHz}$ was investigated by measurement of the $H_{\beta}$ line. By comparing the Voigt profile of $H_{\beta}$ and measured line, the $\Delta \lambda_{S}$ was estimated as $0.107 \mathrm{~nm}$ resulting in an electron density of $3.9 \times 10^{14} \mathrm{~cm}^{-3}$. Electron density in a double-power electrode dielectric barrier discharge plasma jet operating in argon was studied in [121]. A density of $10^{14} \mathrm{~cm}^{-3}$ was measured, which is almost on the limit of theoretical calculations without consideration of fine structure of the $H_{\beta}$ line. In other works various different atmospheric pressure plasma sources with relatively high electron density were successfully studied by the measurements of Stark broadening of hydrogen lines [122-126]. In [126] it was shown that the profile of the $H_{\beta}$ line can be strongly affected by the high electrical field in micro-plasma jets. At electron densities of about $5 \times 10^{14} \mathrm{~cm}^{-3}$ a high electric field of 
Table 2. Fitting equations for estimation of electron density based on measured Stark broadening $\Delta \lambda_{S}$ of hydrogen Blamer series, He I and Ar I lines.

\begin{tabular}{|c|c|c|c|}
\hline Line, $\lambda_{0}[\mathrm{~nm}]$ & $n_{e}\left[\mathrm{~cm}^{-3}\right]$ & Fitting equation & Ref. \\
\hline$H_{\alpha}$ & $>5 \times 10^{14}$ & $n_{e}=10^{17} \times\left(\Delta \lambda_{s}^{A} / 1.098\right)^{1.47135}, n_{e}$ in $\mathrm{cm}^{-3}, \Delta \lambda_{s}^{A}$ in $\mathrm{nm}$ & [14] \\
\hline$H_{\beta}$ & $1.5 \times 10^{14} \div 30 \times 10^{16}$ & $n_{e}=10^{16} \times\left(\Delta \lambda_{S} / 0.94666\right)^{1.49}, n_{e}$ in $\mathrm{cm}^{-3}, \Delta \lambda_{S}$ in $\mathrm{nm}$ & [13] \\
\hline$H_{\beta}$ & $0.03 \div 3.16 \times 10^{16}$ & $\begin{aligned} \log \left(n_{e}\right)= & 22.578+1.478 \times \log \left(\Delta \lambda_{S}\right)-0.144 \times \log \left(\Delta \lambda_{S}\right)^{2} \\
& -0.1265 \times \log \left(T_{e}\right)\end{aligned}$ & [103] \\
\hline & & $n_{e}$ in $\mathrm{m}^{-3}, T_{e} \mathrm{~K}, \Delta \lambda_{S}$ in $\mathrm{nm}$ & \\
\hline$H_{\beta}$ & $>10^{14}$ & $n_{e}=10^{17} \times\left(\Delta \lambda_{S} / 4.8\right)^{1.46808}, n_{e}$ in $\mathrm{cm}^{-3}, \Delta \lambda_{S}$ in $\mathrm{nm}$ & [14] \\
\hline$H_{\gamma}$ & $>10^{14}$ & $n_{e}=10^{17} \times\left(\Delta \lambda_{s}^{A} / 4.668\right)^{1.45826}, n_{e}$ in $\mathrm{cm}^{-3}, \Delta \lambda_{s}^{A}$ in $\mathrm{nm}$ & [14] \\
\hline He I, 318.8 & $1.25 \div 6.22 \times 10^{16}$ & $\begin{array}{l}\Delta \lambda_{S}=n_{e}\left[0.67+4.95 \times 10^{-7} n_{e}^{\frac{1}{4}}\left(1-4.81 \times 10^{-5} n_{e}^{\frac{1}{6}}\right)\right], n_{e} \\
\text { in } \mathrm{m}^{-3}, \Delta \lambda_{S} \text { in nm }\end{array}$ & [114] \\
\hline $\begin{array}{l}\text { He I, } 4 \text { lines for transitions } \\
\text { between } n=3, n=4\end{array}$ & $10^{15} \div 10^{18}$ & $\begin{array}{l}\log _{10}\left(n_{e}\right)=a+b \times\left(\sqrt{T_{e} / T_{c}}+\sqrt{T_{c} / T_{e}}+\sqrt{T_{e} / T_{m} \mu}\right)+p \log _{10}\left(\Delta \lambda_{S}\right), \\
n_{e} \text { in }\left(\mathrm{m}^{-3}\right), \mu \text { is reduced mass in units of proton mass. }\end{array}$ & [115] \\
\hline He I, 501.6 & $1 \div 16.5 \times 10^{16}$ & $\begin{array}{l}\ln \left(\Delta \lambda_{S}\right)=-38.99+1.08 \times \ln \left(n_{e}\right)-0.12 \times \ln \left(T_{e}\right), n_{e} \text { in } \\
\mathrm{cm}^{-3}, \Delta \lambda_{S} \text { in } \AA, T_{e} \text { in K }\end{array}$ & [116] \\
\hline He I, 667.8 & $1 \div 10 \times 10^{16}$ & $\begin{array}{l}\ln \left(\Delta \lambda_{S}\right)=-34.90+1.04 \times \ln \left(n_{e}\right)-0.35 \times \ln \left(T_{e}\right), n_{e} \text { in } \\
\mathrm{cm}^{-3}, \Delta \lambda_{S} \text { in } \AA, T_{e} \text { in } \mathrm{K}\end{array}$ & [116] \\
\hline He I, 471.3 & $0.6 \div 13 \times 10^{16}$ & $\begin{array}{l}\ln \left(\Delta \lambda_{S}\right)=-39.97+1.05 \times \ln \left(n_{e}\right)+0.13 \times \ln \left(T_{e}\right), n_{e} \text { in } \\
\mathrm{cm}^{-3}, \Delta \lambda_{S} \text { in } \AA, T_{e} \text { in } \mathrm{K}\end{array}$ & [116] \\
\hline He I, 728.1 & $1.5 \div 14.5 \times 10^{16}$ & $\ln \left(\Delta \lambda_{S}\right)=8.96 \times 10^{-17} n_{e}+0.282, n_{e}$ in $\mathrm{cm}^{-3}, \Delta \lambda_{S}$ in $\AA$ & [116] \\
\hline $\begin{array}{l}\text { Ar I, } 7 \text { lines of } 4 s-4 p \text { and } \\
4 s-4 p \text { transitions }\end{array}$ & $2 \div 10 \times 10^{16}$ & $\Delta \lambda_{S}$ is presented as a function of $n_{e}$ and $T_{e}$ in Tables & [117] \\
\hline Ar I, 696.5 & $3 \times 10^{15} \div 2 \times 10^{17}$ & $\Delta \lambda_{S}=0.00537 \times n_{e}\left[1+5.6 \times 10^{-6} n_{e}^{\frac{1}{4}}\left(1-0.068 T_{e} n_{e}^{\frac{1}{6}}\right)\right]$ & [118] \\
\hline Ar I, 430.0 & $1.1 \div 11 \times 10^{16}$ & $\begin{array}{l}n_{e} \text { in } \mathrm{cm}^{-3}, \Delta \lambda_{S} \text { in } \mathrm{nm}, T_{e} \text { in } \mathrm{K} \\
\ln \left(n_{e}\right)=44.232+0.992 \times \ln \left(\Delta \lambda_{S}\right)-0.612 \ln \left(T_{e}\right), n_{e} \text { in } \\
\mathrm{cm}^{-3}, \Delta \lambda_{S} \text { in } \mathrm{nm}, T_{e} \text { in } \mathrm{K}\end{array}$ & [119] \\
\hline
\end{tabular}

Parameters $a, b, T_{c}, T_{m}, p$ for He lines $388.9,587.6,706.5,728.1 \mathrm{~nm}$ are presented in table 3 in according with fitting results of [115].

Table 3. Fitting parameters $a, b, T_{c}, T_{m}$, and $p$ for He I lines 388.9, 587.6, 706.5, 728.1 nm. Publishing copyright permission of Astronomy and Astrophysics [115].

\begin{tabular}{llllrl}
\hline Line, $\mathrm{nm}$ & $p$ & $a$ & $b$ & $T_{c}$ & $T_{m}$ \\
\hline He I 728.1 & 0.973601 & 22.786715 & 0.118086 & 42124 & 539893 \\
He I 388.9 & 0.980886 & 23.411952 & 0.089655 & 18807 & 293037 \\
He I 587.6 & 0.988943 & 23.287156 & 0.083064 & 11648 & 132390 \\
He I 706.5 & 0.983379 & 23.093489 & 0.076227 & 100848 & 171832 \\
\hline
\end{tabular}

$15 \mathrm{kV} \mathrm{cm}^{-1}$ can lead to the formation of a dip in the center of the line, that wouldn't appear naturally at such low densities, shown in figure 5, adding further complication to the analysis of the Stark broadening component.

Another application of Stark broadening of hydrogenated lines in diagnostics of APPJs was explored in [127]. In a plasma jet operating at $27.12 \mathrm{MHz}$ at $8 \mathrm{~W}$ in Ar the broadening of Balmer $\beta$ and Balmer $\gamma$ lines was measured. The so called cross-point method was used to evaluate electron density and electron temperature simultaneously. The approach is based on dependence of the Stark broadening of both lines differently on the electron density and the temperature. In a quasi-static approximation for the ions and impact for the electrons $[11,12]$ the relation can be presented as:

$$
n_{e}=3.75\left(\frac{\Delta \lambda_{S}^{\beta, \gamma} \times 10^{10}}{2 \pi \alpha_{1 / 2}^{q}\left(n_{e}, T_{e}\right)}\right)^{1.5}
$$

where $n_{e}$ is in $\mathrm{cm}^{-3}$ and $\Delta \lambda_{S}^{\beta, \gamma}$ is the Stark broadening in $\mathrm{nm}$ for the Balmer $\beta$ and $\gamma$ lines, respectively. The parameter $\alpha_{1 / 2}^{q}\left(n_{e}, T_{e}\right)$ depends on the electron density and temperature and can be found in Griem's tables for different lines [12]. The cross-section of equation (20) for Balmer $\beta$ and $\gamma$ lines in coordinate $n_{e}, T_{e}$ allows estimation of both values. The graphical solution of equation (20) with the crossing-point method is shown in figure 6. The cross-point approach used in [127] leads to an electron concentration of 2.4 to $3.0 \times 10^{14} \mathrm{~cm}^{-3}$ and an electron temperature between 20000 and $30000 \mathrm{~K}$. 

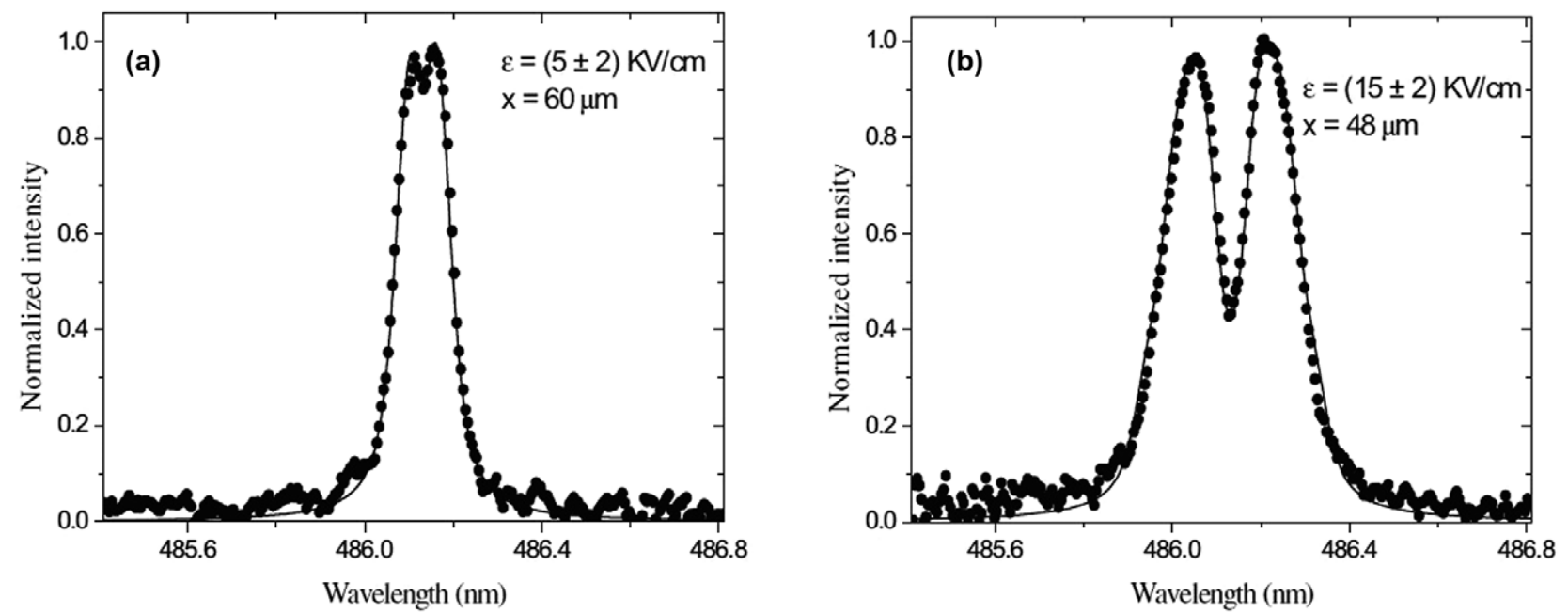

Figure 5. Split of $H_{\beta}$ line in dc micro-plasma jet due to high value of electrical field. Springer copyright permission 2014 [126].

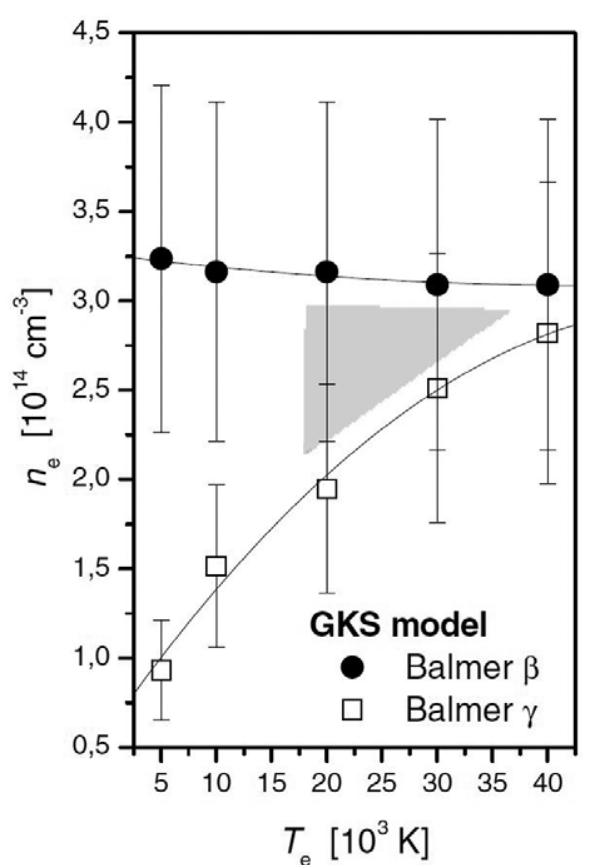

Figure 6. Application of cross-point method for estimation of electron density and temperature from the Stark broadening of $H_{\beta}$ and $H_{\gamma}$ lines. Springer copyright permission 2014 [127].

Unfortunately, the sensitivity of the cross-point method is not very high and $T_{e}$ can only be determined with a large uncertainty of about $0.5 \mathrm{eV}$. The Standard Theory of Stark broadening [12] used in this calculation is an old one with a high inaccuracy in the $H_{\gamma}$ calculation due to its treatment of ion dynamics, which are strongly dependent on the gas temperature. There are also several works, for example works by Sola et al [128, 129], that use the crossing-point method from the line widths in [14], but even using these results the crossing point method is not very accurate, this is due to inaccuracies in the experimental measurements, inaccuracies in the calculated widths (around 5-7\%) and to the flat dependence of the $H_{\beta}$ width with the temperature.

As already mentioned, for many atmospheric pressure plasmas hydrogen appearing as an impurity due to air diffusion in to the discharge zone is not a primary choice for measurements of electron density. Other non-hydrogenated lines have been used in many works where measurements of hydrogen Balmer lines have met certain difficulties. An atmospheric pressure Ar plasma torch generated with a hollow needle-to-plate dielectric barrier discharge was studied in [130]. The electron density estimated from broadening of $H_{\alpha}$ and $\operatorname{Ar} I(696.54 \mathrm{~nm})$ lines was about $1.0 \times 10^{15}$ and $3.78 \times 10^{15} \mathrm{~cm}^{-3}$, respectively. The difference between the two values was explained by the origin of the $H_{\alpha}$ line coming from the dissociation and excitation of $\mathrm{H}_{2} \mathrm{O}$ at the plasma torch surface, whereas Ar I emission was mostly from the inside of the plasma where the density is expected to be higher. Subsequently, a similar source was studied in [131] using the profiles of both $H_{\alpha}$ and $H_{\beta}$ resulting in a self-consistent electron density of about $5.8 \times 10^{14}$ to $6.0 \times 10^{14} \mathrm{~cm}^{-3}$. In [132] the non-hydrogenated Ar I line at $696.54 \mathrm{~nm}$ was used to estimate the electron density in a $23 \mathrm{kHz}$ atmosphericpressure dielectric barrier discharge working with Ar. It was found that the Stark broadening $\Delta \lambda_{S}$ was about $0.00337 \mathrm{~nm}$ which corresponds to an electron density $3.05 \times 10^{15} \mathrm{~cm}^{-3}$ in the filaments forming the DBD. In principal, the procedure to extract electron density information from non-hydrogenic emission lines is similar to that used for hydrogenic lines as presented in figure 4. A deconvolution of the emission line profile is required to first separate the Gaussian profile from the Lorentzian profile. Unfortunately, in practice the measurement of $n_{e}$ from non-hydrogenic emission lines can be significantly more problematic than envisaged from the simple procedure detailed above. It is well known that line profiles from many neutral atoms exhibit an asymmetric profile in high electron density plasmas; this significantly complicates the deconvolution procedure yielding high levels of measurement uncertainty. Djurovic and co-workers attribute the asymmetry to the effects of ion broadening and provide a comprehensive procedure to extract $n_{e}$ information from an asymmetric line profile [112].

An additional source of asymmetry in the line profile can occur in plasma sources that are not spatially homogenous. Zhu et al examined a nanosecond pulsed Ar microplasma 

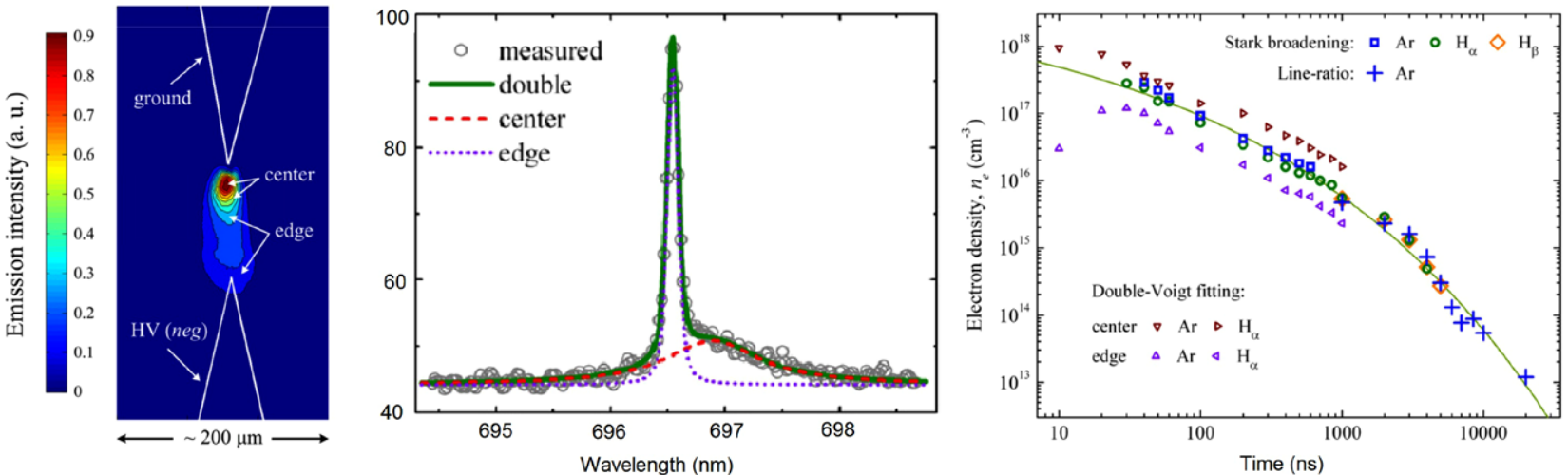

Figure 7. (a) iCCD image of nanosecond pulsed microdischarge (0-10 ns), $(b)$ asymmetric Ar $696.5 \mathrm{~nm}$ line profile, $(c)$ evolution of electron density following application of a nanosecond pulse determined using various methods. IOP Publishing copyright permission 2014 [110].

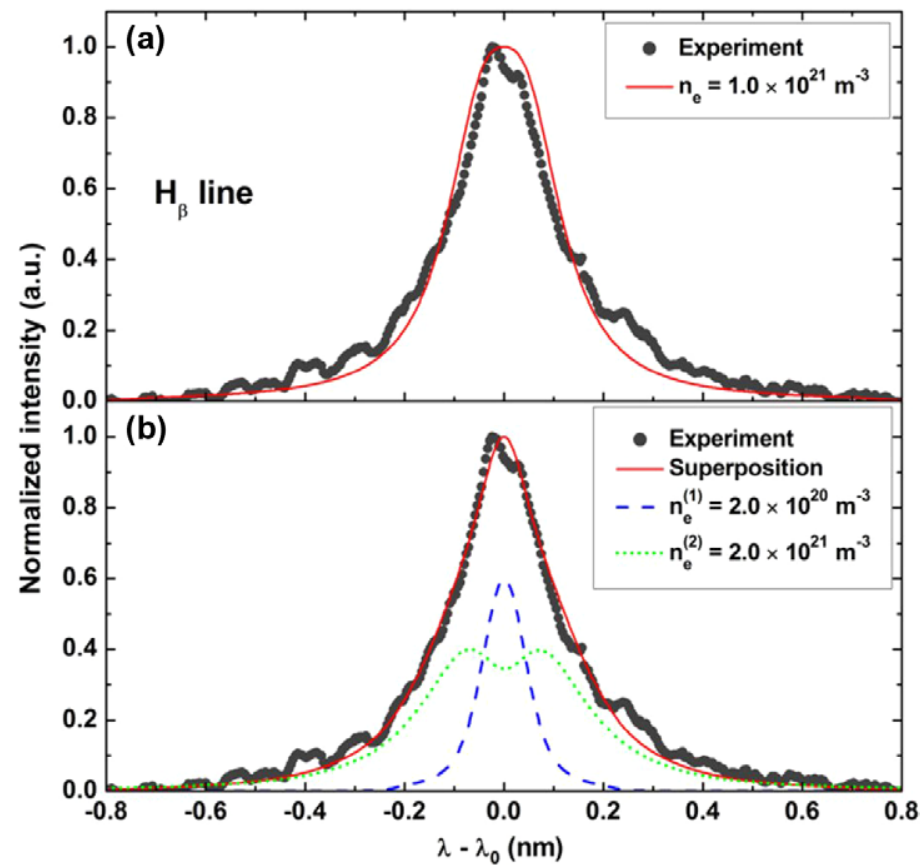

Figure 8. Comparison of single fit of Stark broadening component of $H_{\beta}$ with superposition of two profiles for He discharge working at $15 \mathrm{kV}$ applied voltage. IOP Publishing copyright permission 2014 [134].

[110]; through iCCD imaging they observed an intense central discharge region surrounded by a less intense edge region, shown in figure 7. Spatially averaged optical emission profiles of the Ar $696.5 \mathrm{~nm}$ line obtained during the application of a nanosecond duration voltage pulse showed a considerable level of asymmetry. This asymmetry was attributed to the presence of the two distinct discharge regions, each with considerably different electron densities. Using a double Voigt fitting procedure the temporarily resolved $n_{e}$ in both regions was determined; the results indicated that a peak density of $9.4 \times 10^{17} \mathrm{~cm}^{-3}$ was reached in the central region with a corresponding density of $3.0 \times 10^{16} \mathrm{~cm}^{-3}$ in the edge region. Following application of the nanosecond duration voltage pulse the discharge cooled and a rapid decay in electron density occurred; during the cooling period alternative methods (Line ratio, $H_{\alpha}$ and $H_{\beta}$ broadening) were also applied to measure $n_{e}$ and showed close agreement with the results obtained from the Stark broadening of the $696.5 \mathrm{~nm}$ line in figure 7(c)) [133]. Similar to [133] in [134] distortion of $H_{\beta}$ and He I lines in a pulsed plasma jet working in He has been observed. The theoretical profile was calculated using computer simulation taking into account ion dynamics. The resulting Stark broadening profile consists of two contributions as shown in figure 8 . From the composition of the two profiles, a low electron density of $2.0 \times 10^{16} \mathrm{~cm}^{-3}$ corresponding to the edge of the discharge and high $n_{e}$ of $2.0 \times 10^{17} \mathrm{~cm}^{-3}$ in the core region of the jet have been found.

\section{Low electron density discharges}

Through necessity, plasma sources developed for biomedical applications are typically designed to generate a low-density, low-temperature discharge that is suitable for the direct 


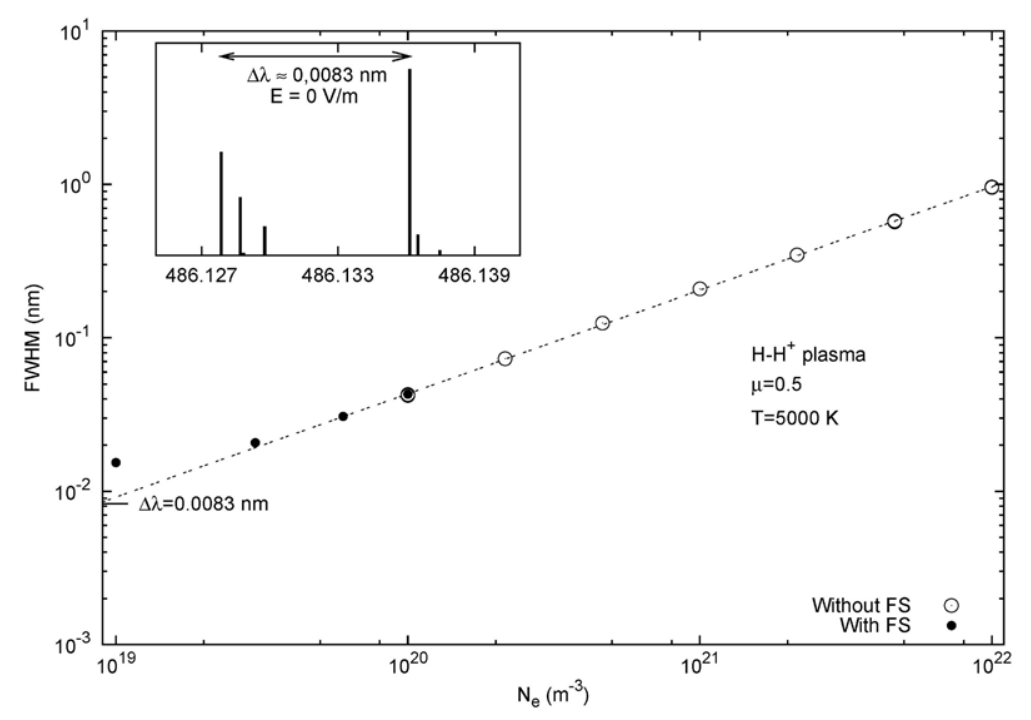

Figure 9. Full width at half maximum of $H_{\beta}$ line. Results of calculations considering fine structure (FS) and without FS are compared. For illustration the inset figure illustrates the fine structure components (the seventh component is hardly seen due to its weak intensity) for an unperturbed atom.

treatment of thermally liable living substrates. Noble gases such as helium and argon are often used combined with various techniques to actively restrict the discharge current, yielding a plasma source that operates close to 'room-temperature' with a low electron density. At low densities, when Stark broadening does not dominate the line broadening, and the Stark width is on the order of the other broadening effects, like the van der Waals or resonance broadening a common technique is to obtain the Stark width from a deconvolution of the experimental profile [91], as discussed previously. Unfortunately, large errors up to an order of magnitude can be induced in the determination of the electron density if inappropriate deconvolution methods are used, as demonstrated in [15].

An additional problem appears in some investigations that conduct plasma diagnostics at low densities using hydrogen Balmer lines. If the plasma density is below, approximately $6 \times 10^{14} \mathrm{~cm}^{-3}$ for $H_{\alpha}$ or $4 \times 10^{13} \mathrm{~cm}^{-3}$ for $H_{\beta}$, the separation of the fine structure components of the line is larger than their Stark broadening and correctly calculated line profiles including the fine structure should be used for comparison with the experimental ones. This is not a recent problem [135] and the practical difficulty comes from the lack of calculated tables of line profiles including fine structure; this has pushed different experimentalists to explore some approximate methods to obtain calculated profiles to compare with their results. The first calculation of the Balmer alpha line with fine structure was published in [136] using the MMM model. Later on, results for the same line were obtained using computer simulations $[137,138]$ and a notable improvement in the agreement with experimental profiles at low electron densities was obtained [139]. The only available tables of Stark broadened line profiles calculated taking into account fine structure correspond to the hydrogen Lyman-alpha line [79]. However, many APPJs are working at a very low ionization degree corresponding to the electron density well below the fine structure limit and various attempts have been made in order to extrapolate the available calculation results to $n_{e}<10^{14} \mathrm{~cm}^{-3}$. In [140] an electron density as low as $4.0 \times 10^{13} \mathrm{~cm}^{-3}$ was obtained through measurement of the $H_{\beta}$ line profile in a $\mathrm{He}$ direct current microplasma of $8 \mathrm{~mA}$. Mu-Yang Qian et al [141] used the $H_{\beta}$ line to estimate $n_{e}$ in an $\mathrm{Ar} 30 \mathrm{kHz}$ DBD jet and obtained a low electron density of about $8.9 \times 10^{13} \mathrm{~cm}^{-3}$ with an applied voltage of $7.5 \mathrm{kV}$; this value is below the applicability of the fitting equations from table 2 and tabulated calculation results for the Stark broadening. The atmospheric pressure helium capillary dielectric barrier discharge has been studied in [142] through $H_{\beta}$ line detection and an electron density of $1.4 \times 10^{12} \mathrm{~cm}^{-3}$ has been obtained with following equation:

$$
\Delta \lambda_{S}=4.60 \times\left[n_{e}\left(\mathrm{~cm}^{-3}\right) \times 10^{-17}\right]^{0.681}
$$

which was originally derived in [14] for $n_{e}>10^{14} \mathrm{~cm}^{-3}$.

Obviously, such a low electron density obtained through Stark broadening of the $H_{\beta}$ line without consideration of the fine structure of the line and ion dynamics can be used only as a rough and indicative value and as such requires additional confirmation by alternative methods. Some experimentalists [109, 143] have employed approximate methods to obtain Stark profiles below the fine structure limits. In [143] a correction to the deconvolution procedure has been proposed in order to take into account the fine structure of the $H_{\beta}$ line. The fine structure of $H_{\beta}$ has been convoluted with an instrumental function which was then used in the calculations. The approach used fixed wavelength positions and intensities of the fine structure components of the unperturbed atom (pertuber electric field $E=0$ ) while assuming a Lorentzian shape for each of the line components with line widths linearly extrapolated to lower values of $n_{e}$ from the tables of calculated line shapes without structure [14]. The Stark broadening $\Delta \lambda_{\mathrm{S}}$ was determined and attributed to electron density of $0.5 \times 10^{13} \mathrm{~cm}^{-3}$. A similar procedure was used in [144] for the $H_{\beta}$ line detected 


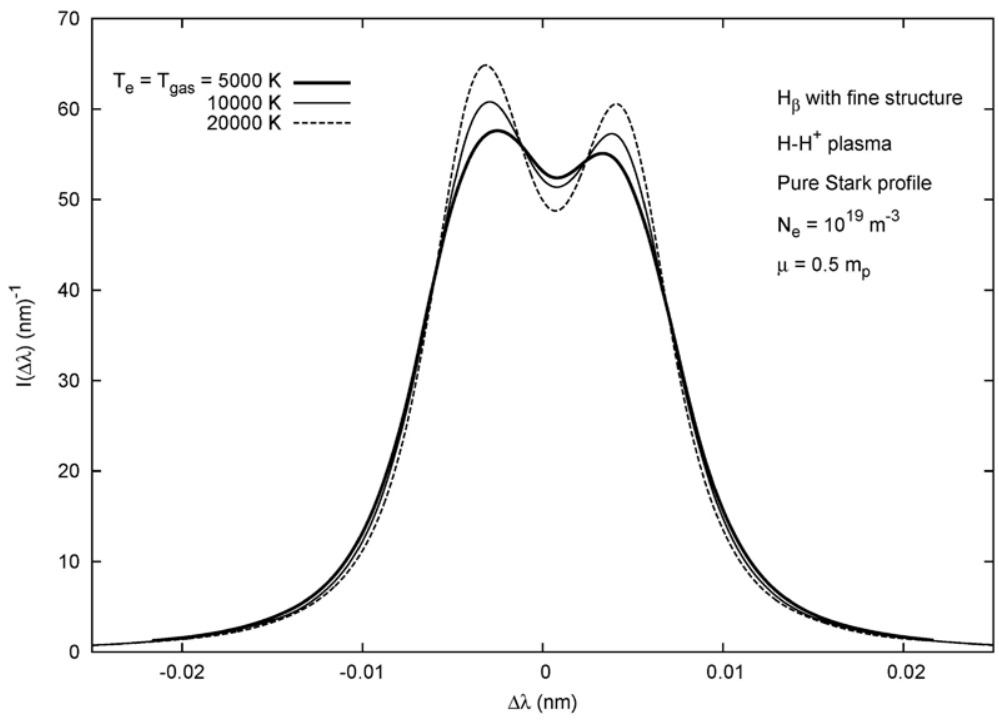

Figure 10. Example of the dependence of the line profile with the plasma temperature showing how the interactions with the heavy perturbers become less effective as they increase their temperature. For comparison, the three profiles are area normalized.

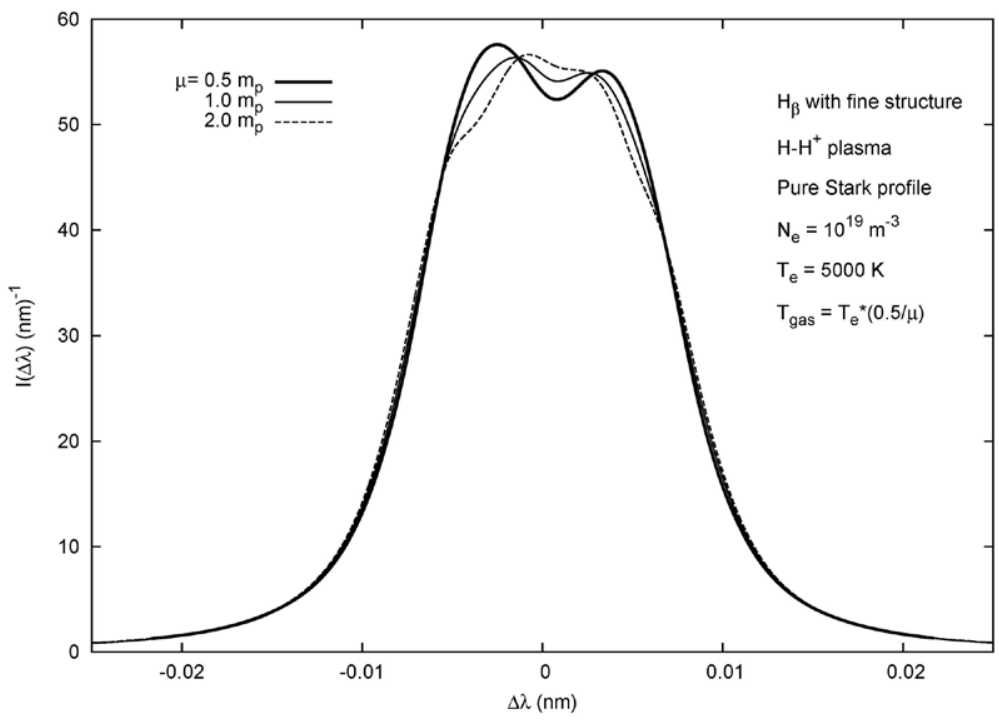

Figure 11. Comparison of the effect of thermal unbalances between electrons and ions in the plasma on the line profile. The three profiles are normalized in area for comparison.

in an RF 11.7 MHz plasma jet in $\mathrm{He}$ and $\mathrm{Ar} / \mathrm{He}$ mixtures with extension of the simulation to low electron density [14]. The effects of fine structure have been considered by fitting the theoretical spectra with a double Lorentzian profile with components separated $8 \mathrm{pm}$ from each other. The procedure has been applied to measure $n_{e}$ at the range of $6 \times 10^{14} \mathrm{~cm}^{-3}>$ $n_{e}>1 \times 10^{13} \mathrm{~cm}^{-3}$. This approach can introduce relatively large errors in $n_{e}$ estimations, as can be seen, for example, in the discussion section of [15] and the only correct procedure would be to use extended, below fine structure limit, tables of line profiles calculated with fine structure.

For an illustration of the effect of fine structure on the Stark broadened Balmer beta line at low densities, some results of the simulated profiles obtained considering fine structure are shown in this work. These profiles were obtained using the simulation technique described in [14]. The line profiles were obtained including fine structure of level $n=4$ and $n=2$, as well as the interaction with the plasma through the dipole interaction. In this way the dependence of the positions and intensities of the fine structure components on $n_{e}$, as well as the broadening of the line, is obtained correctly. Figure 9 shows the dependence of the full width at half maximum of the line as the electron density decreases. At high densities the results without fine structure are shown together with the linear dependence of the line width with the electron density. As can be seen, for densities below approximately $4 \times 10^{13} \mathrm{~cm}^{-3}$ the fine structure calculation shows a higher width than the extrapolation of the non-fine structure results, demonstrating much weaker dependence on the electron density. Figures 10 and 11 show different effects of the temperature on the line profile. Figure 10 compares profiles obtained assuming kinetic equilibrium in the plasma, $T_{e}=T_{\mathrm{gas}}$, but with different temperatures. As can be seen, as temperature increases the heavy perturbers move faster and their broadening decreases, 


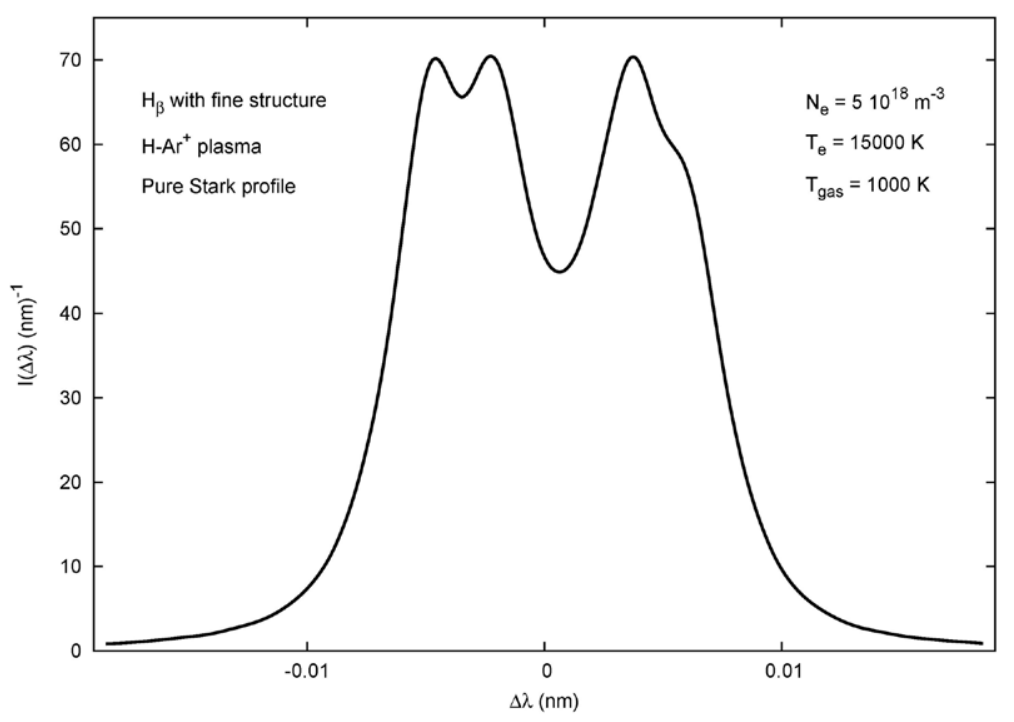

Figure 12. Stark broadened profile of Balmer beta line for a low density $\left(n_{e}=5 \times 10^{12} \mathrm{~cm}^{-3}\right)$. At this low density, the Stark width of the components is small enough, so that the fine structure of the line begins to appear more clearly.

which is a typical behavior of the impact regime, and the line becomes narrower. Figure 11 shows the effect of differences in temperatures between electrons and ions in the plasma, which is kinetically unbalanced. For this, different reduced masses $\mu$ were considered in the simulation [145]. As can be seen, as the ion temperature decreases, they begin to show a less dynamic behavior affecting the line shape. Finally, figure 12 shows the pure Stark profile at a lower electron density, close to some of the experimental conditions in [143]. As the electron density decreases, the Stark width of each component becomes of the order of, or smaller, than the separation between components for that density, and a higher structure of the line is observed.

\section{Summary and conclusions}

This review paper is focused on the analysis of electron density by Stark broadening in atmospheric pressure discharges. Two particular cases of atmospheric pressure plasmas relevant to biomedical applications are considered: high ionization degree discharges with $n_{e}>10^{14} \mathrm{~cm}^{-3}$ and low ionization degree discharges with $n_{e}<10^{14} \mathrm{~cm}^{-3}$. The use of hydrogen Balmer series lines is proposed as the most reliable way to estimate electron densities in the range $1 \times 10^{14} \mathrm{~cm}^{-3}<n_{e}<10^{16} \mathrm{~cm}^{-3}$ where the effect of fine structure splitting of line components does not play an important role. The simple procedure to estimate Stark broadening by fitting of the experimental profile of the line with a Voigt function and deconvolution of Doppler, van der Waals, resonance and self-absorption is discussed including fitting equations for the estimation of the electron density. In high electron density APPJs the use of non-hydrogenic emission lines instead of hydrogen Balmer series to determine $n_{e}$ is proposed as both convenient and highly attractive. The principal of electron density measurement from such lines is in theory very similar to that undertaken when considering hydrogenic lines; however, great care must be taken when adopting the approach. Non-hydrogenic lines are considerably less sensitive than their hydrogenic counterparts; in essence, this limits the applicability of the approach to discharges where the $n_{e}$ is expected to exceed $10^{16} \mathrm{~cm}^{-3}$, conditions that are consummate with atmospheric pressure micro-plasma discharges. In practice, a high degree of asymmetry is observed in the experimental line profiles and the procedure to extract an accurate measure of $n_{e}$ from such profiles is complex, demanding an accurate knowledge of the other broadening mechanisms and an appreciation of the electron temperature in the discharge.

In low electron density plasmas with $n_{e}<10^{14} \mathrm{~cm}^{-3}$ only the hydrogen Balmer lines can be used due to the strong Stark effect. The fine structure of lines and ion dynamics strongly affects the Stark contribution. New fine structure calculations of $H_{\beta}$ lines demonstrate that for densities below $4 \times 10^{13} \mathrm{~cm}^{-3}$ the Stark contribution has higher width than the extrapolation of the non fine structure results, showing much weaker dependence on the electron density. At the lower density of $n_{e}=5 \times 10^{12} \mathrm{~cm}^{-3}$ the Stark width of the components in the Balmer beta line are small enough, so that the fine structure of the line begins to appear. Additionally, thermal imbalances between electrons and ions in the plasma has a pronounced influence on the line profile. The only good practice at such low electron density is the calculation of the Stark component and comparison with experimental results. The use of the widespread practice involving the extrapolation of Stark broadening tables calculated for $n_{e}>10^{14} \mathrm{~cm}^{-3}$ to lower electron density can lead to serious misinterpretation of the experimental results and has to be used with great care after proper validation with independent methods like laser Thomson scattering or heterodyne interferometry.

\section{References}

[1] Laroussi M and Akan T 2007 Plasma Process. Polym. $4777-88$

[2] Schutze A, Jeong J Y, Babayan S E, Park J, Selwyn G S and Hicks R F 1998 IEEE Trans. Plasma Sci. 26 1685-92 
[3] Lu X, Laroussi M and Puech V 2012 Plasma Sources Sci. Technol. 21034005

[4] Sands B L, Ganguly B N and Tachibana K 2008 Appl. Phys. Lett. 92151503

[5] Stonies R, Schermer S, Voges E and Broekaert J A C 2004 Plasma Sources Sci. Technol. 13604

[6] Walsh J L, Iza F, Janson N B, Law V J and Kong M G 2010 J. Phys. D: Appl. Phys. 43075201

[7] Van Gaens W and Bogaerts A 2013 J. Phys. D: Appl. Phys. 46275201

[8] Becker K H, Schoenbach K H and Eden J G 2006 J. Phys. D: Appl. Phys. 39 R55-70

[9] Iza F, Kim G J, Lee S M, Lee J K, Walsh J L, Zhang Y T and Kong M G 2008 Plasma Process. Polym. 5 322-44

[10] Hopwood J, Hoskinson A R and Gregorio J 2014 Plasma Sources Sci. Technol. 23064002

[11] Griem H R 1964 Plasma Spectroscopy (New York: McGraw-Hill)

[12] Griem H R 1974 Spectral Line Broadening by Plasmas (New York: Academic)

[13] Wiese W L, Kelleher D E and Paquette D R 1972 Phys. Rev. A 6 1132-53

[14] Gigosos M A, Gonzalez M A and Cardenoso V 2003 Spectrochim. Acta B 581489

[15] Konjević N, Ivković M and Sakan N 2012 Spectrochim. Acta B 76 16-26

[16] Langmuir I and Mott-Smith H M 1923 Gen. Elec. Rev. 26731

[17] Tichy M, Sícha M, David P and David T 1994 Contrib. Plasma Phys. 3459

[18] Tichy M 2009 Plasma Sources Sci. Technol. 18014009

[19] Yang G, Cronin P, Heberlein J V and Pfender E 2006 J. Phys. D: Appl. Phys. 392764

[20] Raizer Y P 1991 Gas Discharge Physics (Berlin: Springer)

[21] Sarani A, Nikiforov A Y and Leys C 2010 Phys. Plasmas 17063504

[22] Keller S, Bibinov N, Neugebauer A and Awakowicz P 2013 J. Phys. D: Appl. Phys. 46025402

[23] Laroussi M and Lu X P 2005 Appl. Phys. Lett. 87113902

[24] Walsh J L and Gregorio M G 2007 Appl. Phys. Lett. 91221502

[25] Yonemori S, Nakagawa Y, Ono R and Oda T 2012 J. Phys. D: Appl. Phys. $\mathbf{4 5} 225202$

[26] Dyatko N A, Kochetov I V, Napartovich A P and Sukharev A G EEDF: the software package for calculations of the electron energy distribution function in gas mixtures: www.lxcat.laplace.univ-tlse.fr/software/EEDF/

[27] Leipold F, Stark R H, El-Habachi A and Schoenbach K H 2000 J. Phys. D: Appl. Phys. 33 2268-73

[28] Choi J-Y, Takano N, Urabe K and Tachibana K 2009 Plasma Sources Sci. Technol. 18035013

[29] Urabe K, Sakai O and Tachibana K 2011 J. Phys. D: Appl. Phys. 44115203

[30] Burm K T 2005 Plasma Phys. 71 379-88

[31] Batal A, Jarosz J and Mermet J M 1981 Spectrochim. Acta B 36 983-92

[32] Restrepo E and Devia A 2004 J. Vac. Sci. Technol. A 22 377-82

[33] Iordanova E, de Vries N, Guillemier M and van der Mullen J J A M 2008 J. Phys. D: Appl. Phys. 41015208

[34] Phelps A V and Petroviĉ Z L 1999 Plasma Sources Sci. Technol. 8 R21-44

[35] Iordanova E, Palomares J M, Gamero A, Sola A and van der Mullen J J A M 2009 J. Phys. D: Appl. Phys. 42155208

[36] Kempkens H and Uhlenbusch J 2000 Plasma Sources Sci. Technol. 9 492-506

[37] Evans D E and Katzenstein J 1969 Rep. Prog. Phys. 32207
[38] Peacock N J, Robinson D C, Forrest M J, Wilcock P D and Sannikov V V 1969 Nat. London 224488

[39] Belostotskiy S G, Khandelwal R, Wang Q, Donnelly V M, Economou D J and Sadeghi N 2008 Appl. Phys. Lett. 92221507

[40] van Gessel A F H, Carbone E A D, Bruggeman P J and van der Mullen J J A M 2012 Plasma Sources Sci. Technol. 21015003

[41] Shneider M N and Miles R B 2005 J. Appl. Phys. 98033301

[42] Shashurin A, Shneider M N, Dogariu A, Miles R B and Keidar M 2010 Appl. Phys. Lett. 96171502

[43] Keidar M, Shashurin A, Volotskova O, Stepp M A, Srinivasan P, Sandler A and Trink B 2013 Phys. Plasmas 20057101

[44] Zhang Z, Shneider M N and Miles R B 2007 Phys. Rev. Lett. 98265005

[45] Helbig V and Nick K-P 1981 J. Phys. B At. Mol. Opt. Phys. 14 3573-83

[46] Landau L D and Lifshits E M 1965 Quantum Mechanics Non-relativistic Theory: Transl. from the Russian, ed J B Sykes and J S Bell 2nd edn (Oxford: Pergamon)

[47] Bethe H A and Salpeter E E 1977 Quantum Mechanics Of One-And Two-Electron Atoms vol 168 (New York: Plenum)

[48] Baranger M and Mozer B 1959 Phys. Rev. 115 521-5

[49] Barbés A, Gigosos M A and González M A 2001 J. Quant. Spectrosc. Radiat. Transfer $\mathbf{6 8} 679-88$

[50] Vidal C R, Cooper J and Smith E W 1973 Astrophys. J. Suppl. Ser. 25 37-136

[51] Lemke M 1997 Astron. Astrophys. Suppl. Ser. 122 285-92

[52] Dufty J W 1970 Phys. Rev. A 2 534-41

[53] Kelleher D E and Wiese W L 1973 Phys. Rev. Lett. 31 1431-4

[54] Wiese W L, Kelleher D E and Helbig V 1975 Phys. Rev. A 11 1854-64

[55] Grützmacher K and Wende B 1977 Phys. Rev. A 16 243-46

[56] Grützmacher K and Wende B 1978 Phys. Rev. A 18 2140-9

[57] Ferri S et al 2014 Atoms 2 299-318

[58] Frisch U and Brissaud A 1971 J. Quant. Spectrosc. Radiat. Transfer 11 1753-66

[59] Brissaud A and Frisch U 1971 J. Quant. Spectrosc. Radiat. Transfer 11 1767-83

[60] Brissaud A and Frisch U 1974 J. Math. Phys. 15 524-34

[61] Seidel J 1977 Z. Naturforsch. A 32 1195-206

[62] Seidel J 1977 Z. Naturforsch. A 32 1207-14

[63] Stehlé C and Hutcheon R 1999 Astron. Astrophys. Suppl. Ser. 140 93-8

[64] Stehlé C and Fouquet S 2010 Int. J. Spectrosc. 2010506346

[65] Smith E W, Talin B and Cooper J 1981 J. Quant. Spectrosc. Radiat. Transfer 26 229-42

[66] Stamm R and Voslamber D 1979 J. Quant. Spectrosc. Radiat. Transfer 22 599-609

[67] Fussmann G 1975 J. Quant. Spectrosc. Radiat. Transfer $15791-809$

[68] Seidel J and Stamm R 1982 J. Quant. Spectrosc. Radiat. Transfer 27 499-503

[69] Gigosos M A, Cardenoso V and Torres F 1986 J. Phys. B: At. Mol. Phys. 19 3027-33

[70] Hegerfeldt G C and Kesting V 1988 Phys. Rev. A 37 1488-96

[71] Calisti A, Stamm R and Talin B 1988 Phys. Rev. A 38 4883-6

[72] Frerichs M R 1989 Z. Phys. D 11 315-21

[73] Halenka J and Olchawa W 1996 J. Quant. Spectrosc. Radiat. Transfer 56 17-28

[74] Alexiou S and Leboucher-Dalimier E 1999 Phys. Rev. E $603436-8$

[75] Sorge S, Günter S and Röpke G 1999 J. Phys. B At. Mol. Phys. 32 675-81

[76] Stambulchik E 2002 PhD thesis Ann Arbor 1050 48106-1346

[77] Gomez Th 2013 PhD thesis University Texas of Austin

[78] Žikić R, Gigosos M A, Ivković M, Gonzalez M A and Konjević N 2002 Spectrochim. Acta B 57 987-98 
[79] González M Á and Gigosos M A 2000 Astron. Astrophys. Suppl. Ser. 145 491-94

[80] Gigosos M A and González M Á 2009 Astron. Astrophys. $503293-9$

[81] Lara N, González M Á and Gigosos M A 2012 Astron. Astrophys. 542 A:75-A:82

[82] Stamm R, Botzanowski Y, Kaftandjian V P, Talin B and Smith E W 1984 Phys. Rev. Lett. 25 2217-20

[83] Stambulchik E and Maron Y 2010 High Energy Density Phys. 6 9-14

[84] Talin B, Calisti A, Godbert L, Stamm R, Lee R W and Klein L 1995 Phys. Rev. A 51 1918-28

[85] Calisti A, Godbert L, Stamm R and Talin B 1994 J. Quant. Spectrosc. Radiat. Transfer 51 59-64

[86] Calisti A, Mosse C, Koubiti M, Stamm R and Talin B 1995 J. Quant. Spectrosc. Radiat. Transfer 54 89-95

[87] Mossé C, Calisti A, Stamm R, Talin B, Lee R and Klein L 1999 Phys. Rev. A 60 1005-14

[88] Godbert-Mouret L, Rosato J, Capes H, Marandet Y, Ferri S, Koubiti M, Stamm R, González M and Gigosos M 2009 High Energy Density Phys. 5 162-5

[89] Ferri S, Calisti A, Mossé C, Mouret L, Talin B, Gigosos M A, González M A and Lisitsa V 2011 Phys. Rev. E 84026407

[90] Stambulchik E and Maron Y 2013 Phys. Rev. E 87053108

[91] Konjević N 1999 Phys. Rep. 316 339-401

[92] Ivković M, Jovićević S and Konjević N 2004 Spectrochim. Acta B 59 591-605

[93] Djurović S and Konjević N 2009 Plasma Sources Sci. Technol. 18 035011-9

[94] Konjević N, Ivković M and Jovićević S 2010 Spectrochim. Acta B 65 593-602

[95] Gigosos M A and González M Á 2007 J. Quant. Spectrosc. Radiat. Transfer 105 533-5

[96] Voigt Munchen W1912 Sitzber Ak. Wiss. Math-Phys. Kl. 42603

[97] Breene R 1961 The Shift and Shape of Spectral Lines (New York: Pergamon)

[98] Lewis E 1980 Phys. Rep. 581

[99] Peach G 1996 Atomic Molecular and Optical Physics Handbook ed G Drake (Woodbury, NY: AIP)

[100] Petrov G M 2002 J. Quant. Spectrosc. Radiat. Transfer 72 281-7

[101] Wiese W L, Huddlestone R H and Leonard S L 1965 Plasma Diagnostic Techniques (New York: Academic)

[102] Moon H-Y, Herrera K K, Omenetto N, Smith B W and Winefordner J D 2009 Spectrochim. Acta B 64 702-13

[103] Czernichowski A and Chapelle J 1985 J. Quant. Spectrosc. Radiat. Transfer 33 427-36

[104] Ivković M, Gonzalez M A, Jovićević S, Gigosos M A and Konjević N 2010 Spectrochim. Acta B 65 234-40

[105] González M Á, Ivković M, Gigosos M A, Jovićević S, Lara N and Konjević N 2011 J. Phys. D: Appl. Phys. 44194010

[106] Ali A W and Griem H R 1966 Phys. Rev. 144366

[107] Xiong Q, Nikiforov A, Lu X, Britun N, Snyders R and Leys C 2011 J. Phys. D: Appl. Phys. 110073302

[108] Foley H 1946 Phys. Rev. 69616

[109] Laux C O, Spence T G, Kruger C H and Zare R N 2003 Plasma Sources Sci. Technol. 12 125-38

[110] Xi-Ming Z, Walsh J L, Wen-Cong C and Yi-Kang P 2012 J. Phys. D: Appl. Phys. 45295201

[111] Walsh J, Iza F and Kong M G 2010 Eur. Phys. J. D 60 523-30

[112] Djurović S and Konjević N 2009 Plasma Sources Sci. Technol. 18035011

[113] Dimitrijevi M S, Christova M and Sahal-Bréchot S 2007 Phys. Scr. 75 809-9
[114] Peláez R J, González V R, Rodríguez F, Aparicio J A and Mar S 2006 Astron. Astrophys. 453 751-4

[115] Gigosos M A, Djurovic S, Savic I, González-Herrero D, Mijatovic Z and Kobilarov R 2014 Astron. Astrophys. 561 A135

[116] Perez C, de la Rosa I, de Frutos A M and Mar S 1991 Phys. Rev. A 446785

[117] Milosavljevic V, Ellingboe A R and Djeni S 2006 Spectrochim. Acta B 61 81-7

[118] Dong L, Ran J and Mao Z 2005 Appl. Phys. Lett. 86161501

[119] Czernichowski A and Chapelle J 1983 Acta Phys. Pol. A 6367

[120] Cheng C et al 2014 Chin. Phys. B 23075204

[121] Muyang Q, Chunsheng R, Dezhen W, Jialiang Z and Guodong W 2010 J. Appl. Phys. 107063303

[122] Gregorio J, Alves L L, Leroy O, Leprince P and Boisse-Laporte C 2010 Eur. Phys. J. D 60 627-35

[123] Zhu X-M, Chen W-C and Pu Y-K 2008 J. Phys. D: Appl. Phys. 41105212

[124] Zhou Q, Cheng C and Meng Y 2009 Plasma Sci. Technol. 11560

[125] Balcon N, Aanesland A and Boswell R 2007 Plasma Sources Sci. Technol. 16 217-25

[126] Sismanoglu B N, Grigorov K G, Santos R A, Caetano R, Rezende M V O, Hoyer Y D, and Ribas V W 2010 Eur. Phys. J. D 60 479-87

[127] Scheafer J, Sigeneger F, Foest R, Loffhagen D and Weltmann K-D 2010 Eur. Phys. J. D 60 531-8

[128] Torres J, van de Sande M J, van der Mullen J J A M, Gamero A and Sola A 2006 Spectrochim. Acta B 6158

[129] Torres J, Jonkers J, van de Sande M J, van der Mullen J J A M, Gamero A and Sola A 2003 J. Phys. D: Appl. Phys. 36 L55

[130] Dong L-F, Liu W-Y, Yang Y-J, Wang S and Ji Y-F 2011 Acta Phys. Sin. 60045202

[131] Dong L, Ji Y, Liu W, Chen J and Yang Y 2012 IEEE Trans. Plasma Sci. 401701

[132] Dong L, Ran J and Mao Z 2005 Appl. Phys. Lett. 86161501

[133] Huang B-D, Zhu X-M, Takashima K and Pu Y-K 2013 J. Phys. D: Appl. Phys. 46464011

[134] Xiong Q, Nikiforov A, González M A, Leys C and Lu X P 2013 Plasma Sources Sci. Technol. 22 015011-24

[135] Ehrich H and Kelleher D E 1978 Phys. Rev. A 17 1686-9

[136] Stehlé C and Feautrier N 1984 Ann. Phys. 9 697-704

[137] Pastor R, Gigosos M A and González M Á 2001 Spectral Line Shapes 11, 15th ICSLS vol 559 pp 79-81

[138] Olchawa W, Olchawa R and Grabowski B 2004 Eur. Phys. J. D 28 119-24

[139] Bruggeman P, Schram D, Gonzalez M A, Rego R, Kong M G and Leys C 2009 Plasma Sources Sci. Technol. 18025017

[140] Wang Q, Koleva I, Donnelly V M and Economou D J 2005 J. Phys. D: Appl. Phys. 38 1690-7

[141] Qian M-Y, Ren C-S, Wang D-Z, Fan Q-Q, Nie Q-Y, Wen X-Q and Zhang J-L 2012 IEEE Trans. Plasma Sci. $401134-41$

[142] Horvatic V, Muller S, Veza D, Vadla C and Franzke J 2014 J. Anal. At. Spectrom. 29498

[143] Palomares J M, Hübner S, Carbone E A D, de Vries N, van Veldhuizen E M, Sola A, Gamero A and van der Mullen J J A M 2012 Spectrochim. Acta B 73 39-47

[144] Hofmann S, van Gessel A F H, Verreycken T and Bruggeman P 2011 Plasma Sources Sci. Technol. 20065010

[145] Gonzalez M A and Gigosos M A 2009 Plasma Sources Sci. Technol. 18034001 